\title{
Control on the Sphere and Reduced Attitude Stabilization
}

\author{
Francesco Bullo ${ }^{1}$ Richard M. Murray \\ Division of Engineering and Applied Science \\ California Institute of Technology \\ Pasadena, CA 91125 \\ Augusto Sarti \\ Dipartimento di Ingegneria Elettronica \\ Politecnico di Milano \\ Milano, Italy
}

March 28, 1995

\footnotetext{
${ }^{1}$ Current address: California Institute of Technology, Mail Code 104-44, Pasadena, CA 91125. E-mail: bullo@indra.caltech.edu
} 


\begin{abstract}
This paper focuses on a new geometric approach to (fully actuated) control systems on the sphere. The control laws exploit the basic and intuitive notions of geodesic direction and distance between points, and generalize the classical proportional plus derivative feedback (PD) without the need of arbitrary local coordinate charts. The stability analysis relies on an appropriate Lyapunov function, where the notion of distance and its properties are exploited. This methodology is applied to spin-axis stabilization of a spacecraft actuated by only two control torques: discarding the rotation about the unactuated axis, a reduced system is considered whose state is defined on the sphere. For this reduced stabilization problem, the approach allows not only an optimal treatment of the inevitable singularity, but also simplicity, versatility and (coordinate independent) adaptive capabilities.
\end{abstract}




\section{Contents}

1 Introduction 1

2 Geodesic control of systems on the sphere 3

2.1 Regulation on the sphere . . . . . . . . . . . . 4

2.2 Trajectory tracking on the sphere . . . . . . . . 9

3 Spacecraft models with two control torques 11

4 Spacecraft control laws $\quad 13$

4.1 P control laws for the kinematic model . . . . . . . . . . . . 14

4.2 PD control laws for the dynamic model . . . . . . . . . 15

5 Simulations $\quad \mathbf{2 0}$

5.1 Reduced attitude regulation of the dynamic spacecraft . . . 20

5.2 Trajectory tracking on the sphere . . . . . . . . . 25

6 Conclusions $\quad 28$

$\begin{array}{ll}\text { References } & 29\end{array}$

$\begin{array}{ll}\text { A Convergence of PD controllers } & 31\end{array}$

A.1 PD regulator . . . . . . . . . . . . . 31

A.2 PD trajectory tracker . . . . . . . . . . . 33

$\begin{array}{ll}\text { B Convergence of adaptive schemes } & \mathbf{3 7}\end{array}$

B.1 Adaptive regulation of kinematic model . . . . . . . . . 37

B.2 Adaptive regulation of dynamic model . . . . . . . . . 38 


\section{Introduction}

The purpose of this paper is twofold. On one hand we design control laws for fully actuated systems defined on the sphere $\mathbb{S}^{2}$. On the other, we apply these laws to the model of a spacecraft actuated by only two control torques and we give complete solution to a reduced attitude stabilization problem, i.e. we stabilize the spacecraft attitude up to a rotation about the unactuated axis. This problem is of practical importance, since it models for example the failure of an actuator, and is a classic, very instructive issue in nonlinear control theory. Indeed new applications to visual tracking problems (Swain and Stricker 1991) seem to offer new examples of systems on spheres.

Within the vast literature on attitude control, Crouch (1984) shows positive controllability results for the case of three independent control torques and various smooth, stabilizing, control laws have been proposed (Wen and Kreutz-Delgado 1991). The case of only two independent controls is more difficult. Indeed, Byrnes and Isidori (1991) show the non-stabilizability of the system: no smooth feedback control law can locally, asymptotically stabilize the full state of a spacecraft with only two actuators. Both discontinuous (Krishnan, Reyhanoglu, and McClamroch 1994) and smooth timevarying control laws (Walsh, Montgomery, and Sastry 1994; Morin, Samson, Pomet, and Jiang 1994) can overcome this limitation by using ideas from the theory of nonholonomic stabilization, but are limited to the case of gas jet actuators.

Following Byrnes and Isidori (1991) and Tsiotras and Longuski (1994), we employ here a reduced approach: by discarding the rotation about the unactuated axis we come down to stabilizing a two dimensional system. The new reduced system is fully controllable (actuated), in that at each position variable corresponds an independent control, and its state is naturally defined on the sphere $\mathbb{S}^{2}$. Therefore our attention turns to the study of control laws on this manifold. Note that Brockett (1973) introduces a quite complete theory of control systems defined on spheres, in that he discusses controllability, observability and optimal control issues. Here instead, we concern ourselves with the explicit search for control laws. Since the manifold $\mathbb{S}^{2}$ is compact, has no boundary and its Euler characteristic is two, no smooth control law with only one stable equilibrium point exists; therefore we must be satisfied with control laws defined (and stabilizing) on a open dense subset of $\mathbb{S}^{2}$. Following Koditschek (1989) we call such feedback law almost-global.

The main contribution of this paper is a novel, general approach to fully actuated control systems defined on the sphere $\mathbb{S}^{2}$. The novelty is based 
on exploiting the metric properties of the Riemannian manifold $\mathbb{S}^{2}$. For a first order model, our control law exerts an action which has intensity proportional to the distance between the state of the system and the goal and is directed along the geodesic direction connecting these two points. An appropriate Lyapunov function based on the Riemannian notion of distance allows us to prove exponential stability. We then extend this control law to second order models through a standard procedure in the robotics literature: we couple the proportional action with a derivative term, i.e. with a term proportional to the "velocity". Again, the exponential stability of this proportional plus derivative (PD) control is based on the metric properties of $\mathbb{S}^{2}$. Our new approach shows two main advantages. First of all the control laws are coordinate invariant (no arbitrary choice of local chart is necessary) so that they allow us to solve the trajectory tracking problem in a global way. Second of all, the ideas can be generalized in a straightforward manner so that "geodesic" control laws can be designed for more general Riemannian manifolds, see (Bullo and Murray 1994).

Regarding the control of the underactuated spacecraft, we cast the problem into this well-suited framework and we give a complete solution to the reduced stabilization problem. Many differences exist with respect to the approach described in Tsiotras and Longuski (1994). First of all we respect and exploit the (geo)metric properties of the sphere instead of relying on a choice of local coordinates. As a result, our almost-global control laws confine the inevitable singularity as far away as possible from the equilibrium (i.e. at the antipodal point) and sufficient conditions on the initial state of the system are provided in order to confine the closed-loop trajectories away from the singularity (instead of simply assuming this as hypothesis). Additionally the control action remains bounded even for large errors. From a practical viewpoint, our feedback laws allow positive definite matrix gains rather than simple positive constants, and the final expression of the control is somewhat simpler than the one given by Tsiotras and Longuski, where unusual cross terms (position-velocity) are present.

The paper is organized as follows: Section 2 deals with some basic Riemannian notions and with the design of PD control laws on the sphere. In Section 3 reduced attitude stabilization is formulated as a control problem on the sphere. In Section 4 we apply our design techniques to kinematic and dynamic models of a spacecraft. Finally we report some simulations in Section 5 and Section 6 contains a brief discussion. 


\section{Geodesic control of first and second order sys- tems on the sphere}

Our goal is to design optimal, in the sense of geodesic, control laws for fully actuated control systems of first and second order, whose states lie on the manifold $\mathbb{S}^{2}=\left\{x \in \mathbb{R}^{3}:\|x\|=1\right\}$. We start by describing the geometric properties of the sphere and by applying some basic results of Riemannian geometry (Boothby 1975). In the following, denote the standard inner and outer products on $\mathbb{R}^{3}$ by $\langle\cdot, \cdot\rangle$ and $[\cdot, \cdot]$. Let $p$ be a generic point on the sphere and $-p$ the corresponding antipodal point. For all points $p \in \mathbb{S}^{2} \subset \mathbb{R}^{3}$, every tangent vector $X_{p} \in T_{p} \mathbb{S}^{2}$ can be uniquely represented as a vector $X_{p} \in$ $\mathbb{R}^{3}$ such that $X_{p} \perp p$ (using the standard inner product on $\mathbb{R}^{3}$ ) and more generally $T_{p} \mathbb{S}^{2}=\operatorname{span}\{p\}^{\perp}$. The canonical inner product on $\mathbb{R}^{3}$ induces a Riemannian structure on $\mathbb{S}^{2}$ (i.e. an inner product on $T_{p} \mathbb{S}^{2}$ ) in the natural way:

$$
\left\langle X_{p}, Y_{p}\right\rangle_{T_{p} \mathbb{S}^{2}} \triangleq\left\langle X_{p}, Y_{p}\right\rangle \quad \forall X_{p}, Y_{p} \in T_{p} \mathbb{S}^{2} \subset \mathbb{R}^{3} .
$$

The geodesics of this natural metric are great circles and the distance between two generic points $p, q \in \mathbb{S}^{2}$ is the angle between the two directions:

$$
\operatorname{dist}(\mathrm{p}, \mathrm{q})=\arccos \left(\langle\mathrm{p}, \mathrm{q}\rangle_{\mathbb{R}^{3}}\right)
$$

with arccos taking values in $[0, \pi]$. Additionally, provided $p$ and $q$ are neither equal nor opposite, there is a uniquely defined unit vector $Y_{p}^{q} \in T_{p} \mathbb{S}^{2}$ called the geodesic versor that gives the geodesic direction in $p$ towards $q$ :

$$
Y_{p}^{q} \triangleq[\operatorname{vers}([p, q]), p]=\operatorname{vers}\left(q_{\perp}\right),
$$

where $q_{\perp}$ is the component of $q$ orthogonal to $p$ and the operator versor simply means: $\operatorname{vers}(x)=x /\|x\|$. The notion of distance and of geodesic versor are related by a useful extension of Gauss's Lemma:

\section{Lemma 1 (Derivative of distance function)}

Consider a trajectory $p=p(t) \in \mathbb{S}^{2}$, such that $p(t)$ never passes through the fixed points $q$ or $-q$. Then

$$
\frac{d}{d t} \operatorname{dist}(\mathrm{p}(\mathrm{t}), \mathrm{q})=-\left\langle\dot{\mathrm{p}}, \mathrm{Y}_{\mathrm{p}}^{\mathrm{q}}\right\rangle_{\mathrm{T}_{\mathrm{p}} \mathbb{S}^{2}}
$$

Proof. Differentiating the definition in equation (2.1), we have

$$
\begin{aligned}
\frac{d}{d t} \operatorname{dist}(\mathrm{p}, \mathrm{q}) & =\frac{d}{d t} \arccos \left(\langle p, q\rangle_{\mathbb{R}^{3}}\right) \\
& =-\frac{\langle\dot{p}, q\rangle}{\sqrt{1-\langle p, q\rangle^{2}}}=-\frac{\left\langle\dot{p}, q_{\perp}\right\rangle}{\|[p, q]\|}=-\left\langle\dot{p}, Y_{p}^{q}\right\rangle .
\end{aligned}
$$




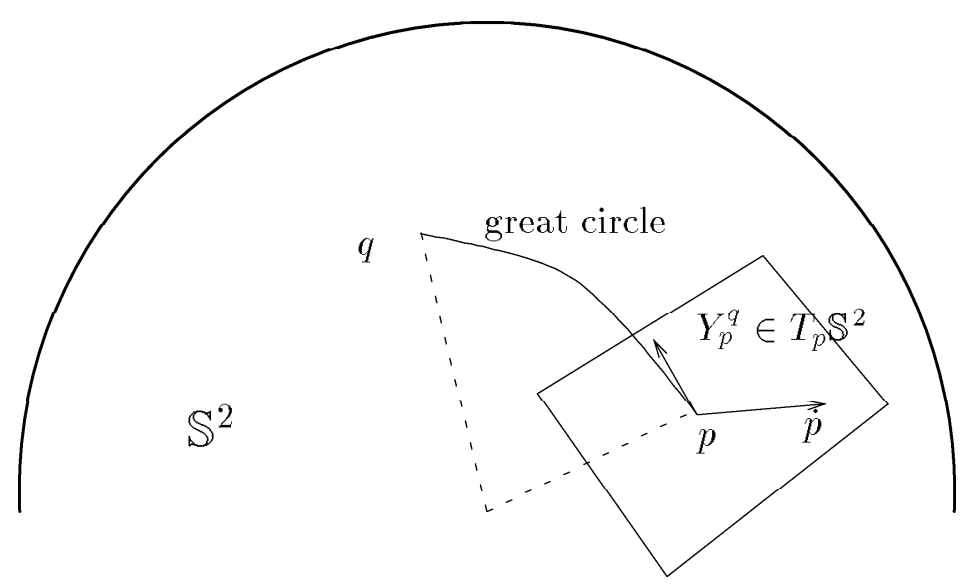

Figure 1: The sphere and its tangent plane.

The terms of Lemma 1 can be intuitively interpreted as follows: the distance between state of the system $p$ and fixed goal $q$ evolves in time only depending on the component of $\dot{p}$ along the geodesic direction $Y_{p}^{q}$. In particular for a trajectory $p(t)$ such that $\operatorname{dist}(\mathrm{p}, \mathrm{q})$ is constant, Lemma 1 reduces to Gauss's Lemma and asserts the orthogonality between $\dot{p}(t)$ and the geodesic versor $Y_{p}^{q}$. Note that the result of Lemma 1 corresponds in $\mathbb{R}^{n}$ to the following equality:

$$
\frac{d}{d t}\|x\|=-\langle\dot{x},-\operatorname{vers}[x]\rangle
$$

where $-\operatorname{vers}[x]$ is the geodesic versor from $x$ to the origin.

\subsection{Regulation on the sphere}

Since we are now able to compute its time derivative, the Riemannian notion of distance appears suitable for stability analysis; in the following let $B_{p}=$ $\left\{X_{p}^{1}, X_{p}^{2}\right\}$ be a smooth orthonormal basis of $T_{p} \mathbb{S}^{2}$. The regulation problem for a fully controllable, first order system defined on the sphere reads: 
Problem 1 (Regulation of first order systems)

Given the system

$$
\dot{p}=X_{p}^{1} v_{1}+X_{p}^{2} v_{2} \quad \in T_{p} \mathbb{S}^{2}
$$

find a control $v=v(p)$ so as to steer asymptotically the state $p \in \mathbb{S}^{2}$ to the fixed goal $q$.

Note that $B_{p}$ cannot be smoothly defined on all $\mathbb{S}^{2}$. A canonical choice in the regulation problem would be

$$
B_{p}=\left\{Y_{p}^{q}, \operatorname{vers}([p, q])\right\},
$$

where the base $B_{p}$ is defined for all $p$ neither equal nor opposite to $q$. More generally a natural choice $B_{p}$ might be suggested by the particular control problem in question; this is the case in Section 4.

To solve this first problem, we generalize the classical proportional control to the manifold $\mathbb{S}^{2}$ as follows: the control action has intensity proportional to the distance between state and goal and is applied along the geodesic direction (connecting state and goal) skewed by a positive definite gain $K_{p}$. This approach is considerably more general rather than choosing a set of local coordinates and applying a proportional control in the local chart. Even the stability analysis for this generalized proportional controller holds in full generality, since the Lyapunov function that proves the exponential convergence relies on the notion of distance

Let $\lambda_{\min }\left(K_{p}\right)$ be the minimum eigenvalue of the positive definite matrix $K_{p}$.

\section{Theorem 2 (Regulation of first order systems)}

Consider the system in equation (2.2). Then the control law

$$
v=\operatorname{dist}(\mathrm{p}, \mathrm{q}) \mathrm{K}_{\mathrm{p}}\left[\begin{array}{c}
\left\langle Y_{p}^{q}, X_{p}^{1}\right\rangle \\
\left\langle Y_{p}^{q}, X_{p}^{2}\right\rangle
\end{array}\right]
$$

exponentially stabilizes the state $p$ at $q$ from any initial condition $p(0) \neq-q$ and with time-constant at least $1 / \lambda_{\min }\left(K_{p}\right)$.

Proof. Consider the candidate Lyapunov function

$$
W \triangleq \frac{1}{2} \operatorname{dist}(\mathrm{p}, \mathrm{q})^{2}
$$


Since $p(0) \neq-q$, Lemma 1 gives

$$
\begin{aligned}
\dot{W} & =\operatorname{dist}(\mathrm{p}, \mathrm{q}) \frac{\mathrm{d}}{\mathrm{dt}} \operatorname{dist}(\mathrm{p}, \mathrm{q}) \\
& =-\operatorname{dist}(\mathrm{p}, \mathrm{q})\left\langle\dot{\mathrm{p}}, \mathrm{Y}_{\mathrm{p}}^{\mathrm{q}}\right\rangle_{\mathrm{T}_{\mathrm{p}} \mathbb{S}^{2}} \\
& =-\operatorname{dist}(\mathrm{p}, \mathrm{q})^{2}\left\langle\mathrm{~K}_{\mathrm{p}}\left[\begin{array}{l}
\left\langle Y_{p}^{q}, X_{p}^{1}\right\rangle \\
\left\langle Y_{p}^{q}, X_{p}^{2}\right\rangle
\end{array}\right],\left[\begin{array}{l}
\left\langle Y_{p}^{q}, X_{p}^{1}\right\rangle \\
\left\langle Y_{p}^{q}, X_{p}^{2}\right\rangle
\end{array}\right]\right\rangle_{\mathbb{R}^{2}} \\
& \leq-2 \lambda_{\min }\left(K_{p}\right) W .
\end{aligned}
$$

Hence the distance between $p(t)$ and $q$ decreases exponentially and the closed-loop system never passes through the singularity point $-q$.

Note that for $p=q$ the geodesic versor $Y_{p}^{p}$ is not defined. This is nota serious problem, since the control law $v$ can be easily prolonged continuously and defined equal to zero at $q: v(q)=0$. Hence the control law in equation (2.3) is smooth on $\mathbb{S}^{2} \backslash\{-q\}$ and has a single exponentially stable equilibrium point $q$. As explained in the introduction, no smooth global control law with only one stable equilibrium point exists on the sphere. Therefore the most we can achieve is an almost-global control law, that is a smooth law defined on a dense subset of the sphere; this is what equation (2.3) gives.

We now consider the more general problem of controlling a system via accelerations (or forces) instead of velocities. The regulation problem for a fully controllable, second order system defined on the sphere reads:

\section{Problem 2 (Regulation of second order systems)}

Given the system

$$
\left\{\begin{array}{l}
\dot{p}=X_{p}^{1} v_{1}+X_{p}^{2} v_{2} \quad X_{p}^{1}, X_{p}^{2} \in T_{p} \mathbb{S}^{2} \\
\dot{v}=u
\end{array}\right.
$$

find a control $u=u(p, \dot{p})$ such as to steer asymptotically the state $p \in \mathbb{S}^{2}$ to the fixed goal $q$.

As typically done in the robotics literature (Murray, Li, and Sastry 1994, Chapter 5, Section 4.3), we now combine proportional and derivative (PD) action. The closed-loop system will behave as a nonlinear spring with a velocity damper and correspondingly the Lyapunov function will be the sum of pseudo-kinetic and pseudo-potential energy terms. With respect to a standard PD controller in local coordinates, the novelty here consists in the form of the proportional action (we have a geodesic spring) and of the corresponding pseudo-potential energy term (Riemannian distance squared). 
Theorem 3 (Regulation of second order systems)

Consider the system in equation (2.4). Given the positive definite matrix gains $K_{p}$ and $K_{d}$, the control law

$$
u=\operatorname{dist}(\mathrm{p}, \mathrm{q}) \mathrm{K}_{\mathrm{p}}\left[\begin{array}{c}
\left\langle Y_{p}^{q}, X_{p}^{1}\right\rangle \\
\left\langle Y_{p}^{q}, X_{p}^{2}\right\rangle
\end{array}\right]-\mathrm{K}_{\mathrm{d}} \mathrm{v},
$$

exponentially stabilizes the equilibrium point $q$ from any initial condition $p(0) \neq-q$ and for all $K_{p}$ and $\dot{p}(0)$ such that

$$
\lambda_{\min }\left(K_{p}\right)>\frac{\|\dot{p}(0)\|^{2}}{\pi^{2}-\operatorname{dist}(\mathrm{p}(0), \mathrm{q})^{2}} .
$$

Proof. Consider the typical candidate Lyapunov function

$$
W \triangleq \frac{1}{2} \operatorname{dist}(\mathrm{p}, \mathrm{q})^{2}+\frac{1}{2}\|\mathrm{v}\|_{\mathrm{K}_{\mathrm{p}}^{-1}}^{2}
$$

Since $p(0) \neq-q$, Lemma 1 holds at least for small $t$, and gives

$$
\begin{aligned}
\dot{W}= & -\operatorname{dist}(\mathrm{p}, \mathrm{q})\left\langle\dot{\mathrm{p}}, \mathrm{Y}_{\mathrm{p}}^{\mathrm{q}}\right\rangle_{\mathrm{T}_{\mathrm{p}} \mathbb{S}^{2}}+\left\langle\mathrm{v}, \mathrm{K}_{\mathrm{p}}^{-1} \mathrm{u}\right\rangle \\
= & -\operatorname{dist}(\mathrm{p}, \mathrm{q})\left\langle\dot{\mathrm{p}}, \mathrm{Y}_{\mathrm{p}}^{\mathrm{q}}\right\rangle_{\mathrm{T}_{\mathrm{p}} \mathbb{S}^{2}-\|\mathrm{v}\|_{\mathrm{K}_{\mathrm{p}}^{-1} \mathrm{~K}_{\mathrm{d}}}^{2}} \\
& +\operatorname{dist}(\mathrm{p}, \mathrm{q})\left\langle\mathrm{v},\left[\begin{array}{c}
\left\langle Y_{p}^{q}, X_{p}^{1}\right\rangle \\
\left\langle Y_{p}^{q}, X_{p}^{2}\right\rangle
\end{array}\right]\right\rangle .
\end{aligned}
$$

Since with respect to the basis $B_{p}$ it holds

$$
\left\langle\dot{p}, Y_{p}^{q}\right\rangle_{T_{p} \mathbb{S}^{2}}=\left\langle\left[\begin{array}{l}
v_{1} \\
v_{2}
\end{array}\right],\left[\begin{array}{c}
\left\langle Y_{p}^{q}, X_{p}^{1}\right\rangle \\
\left\langle Y_{p}^{q}, X_{p}^{2}\right\rangle
\end{array}\right]\right\rangle,
$$

we have

$$
\dot{W}=-\|v\|_{K_{p}^{-1} K_{d}}^{2}
$$

so that, for small $t$, the Lyapunov function in equation (2.5) is decreasing. Because of the condition in equation (2.5), we have

$$
\begin{aligned}
\left.2 W\right|_{t=0} & <\operatorname{dist}(\mathrm{p}(0), \mathrm{q})^{2}+\lambda_{\max }\left(\mathrm{K}_{\mathrm{p}}^{-1}\right)\|\dot{\mathrm{p}}(0)\|^{2} \\
& =\operatorname{dist}(\mathrm{p}(0), \mathrm{q})^{2}+\lambda_{\min }\left(\mathrm{K}_{\mathrm{p}}\right)\|\dot{\mathrm{p}}(0)\|^{2} \\
& <\pi^{2}
\end{aligned}
$$


and, since $\dot{W} \leq 0$,

$$
\forall t>0, \quad \frac{\pi^{2}}{2}>W(t)>\frac{1}{2} \operatorname{dist}(\mathrm{p}(\mathrm{t}), \mathrm{q})^{2}, \quad \Rightarrow \quad \operatorname{dist}(\mathrm{p}(\mathrm{t}), \mathrm{q})<\pi,
$$

proving this way that the state $p$ will never pass through the point $-q$. Therefore Lemma 1 holds for all $t$ and equation (2.6) shows the Lyapunov stability of the closed-loop system.

We can now invoke Lasalle's principle to prove asymptotic stability: the closed-loop trajectories of the system converge asymptotically to the largest invariant set contained in

$$
\Omega\{(p, v): \dot{W}=0\}=\left\{(p, v) \in \mathbb{S}^{2} \times \mathbb{R}^{2}: v=0\right\},
$$

and since in $\Omega$ the dynamic equation of the closed-loop system reduces to

$$
0=\operatorname{dist}(\mathrm{p}, \mathrm{q}) \mathrm{K}_{\mathrm{p}}\left[\begin{array}{l}
\left\langle Y_{p}^{q}, X_{p}^{1}\right\rangle \\
\left\langle Y_{p}^{q}, X_{p}^{2}\right\rangle
\end{array}\right]
$$

then the largest invariant set contained in $\Omega$ is the set $\{(q, 0)\}$.

To prove the exponential stability, we introduce into the Lyapunov function a cross term of the form:

$$
-\epsilon \operatorname{dist}(\mathrm{p}, \mathrm{q})\left\langle\dot{\mathrm{p}}, \mathrm{Y}_{\mathrm{p}}^{\mathrm{q}}\right\rangle_{\mathrm{T}_{\mathrm{p}} \mathbb{S}^{2}}
$$

This procedure is quite standard (see (Wen and Bayard 1988) and (Murray, Li, and Sastry 1994, Chapter 5, Section 4.3)), and we refer to Appendix A, which contains the relative detailed calculations.

Note that the condition expressed in equation (2.5) confines the closed-loop trajectories away from singularity.

As a final result on regulation problems, we show how to generalize the proposed approach to the case of control systems defined on $\mathbb{S}^{n}$. Let $p$ and $q$ be generic points on the sphere $\mathbb{S}^{n} \subset \mathbb{R}^{n+1}$. Even though no outer product is in general defined on $\mathbb{R}^{n}$, we can still define

$$
\operatorname{dist}(p, q) \triangleq \arccos \left(\langle p, q\rangle_{\mathbb{R}^{n}}\right) \text {. }
$$

and

$$
Y_{p}^{q} \triangleq \operatorname{vers}\left[q_{\perp}\right]=\operatorname{vers}[q-\langle q, p\rangle p] \in T_{p} \mathbb{S}^{n}
$$

where $p$ is assumed different from $q$ and $-q$. The extension of Gauss's Lemma still holds; indeed it is easy to prove that

$$
\frac{d}{d t} \operatorname{dist}(\mathrm{p}(\mathrm{t}), \mathrm{q})=-\left\langle\dot{\mathrm{p}}, \mathrm{Y}_{\mathrm{p}}^{\mathrm{q}}\right\rangle_{\mathrm{T}_{\mathrm{p}} \mathbb{S}}
$$


Thus, both our proportional and proportional plus derivative control laws can be easily rewritten for this new case. We shall here give the more general version of Theorem 3 :

Theorem 4 (Regulation of second order systems on $\mathbb{S}^{n}$ )

Let $B_{p}=\left\{X_{p}^{i}, 1 \leq i \leq n\right\}$ be a smooth orthonormal basis of $T_{p} \mathbb{S}^{n}$, and consider the system

$$
\left\{\begin{aligned}
\dot{p} & =v_{1} X_{p}^{1}+\ldots+v_{n} X_{p}^{n} \\
\dot{v} & =u
\end{aligned}\right.
$$

where $v=\left[v_{1} \ldots v_{n}\right]^{T}$. Let $K_{p}$ ad $K_{d}$ be symmetric positive definite gains, then the control law

$$
u=\operatorname{dist}(\mathrm{p}, \mathrm{q}) \mathrm{K}_{\mathrm{p}}\left[\begin{array}{c}
\left\langle Y_{p}^{q}, X_{p}^{1}\right\rangle \\
\vdots \\
\left\langle Y_{p}^{q}, X_{p}^{n}\right\rangle
\end{array}\right]-\mathrm{K}_{\mathrm{d}} \mathrm{v},
$$

exponentially stabilizes the state $p$ at $q$ from any initial condition $p(0) \neq-q$ and for all $K_{p}$ such that

$$
\lambda_{\min }\left(K_{p}\right)>\frac{\|\dot{p}(0)\|^{2}}{\pi^{2}-\operatorname{dist}(\mathrm{p}(0), \mathrm{q})^{2}} .
$$

\subsection{Trajectory tracking on the sphere}

In this subsection we state the trajectory tracking version of our PD control law; let $q \in \mathbb{S}^{2}$ the desired state, $\dot{q}, \ddot{q} \in T_{q} \mathbb{S}^{2}$ be the desired velocity and acceleration. ${ }^{1}$ In the following we will assume $\dot{q}$ bounded. Define $\mathcal{R} \in S O(3)$ as the rotation about $[p, q]$ which maps $p$ to $q$ :

$$
\mathcal{R} \triangleq I_{3}+([p, q] \times)+\frac{1-\cos (\operatorname{dist}(\mathrm{p}, \mathrm{q}))}{\sin (\operatorname{dist}(\mathrm{p}, \mathrm{q}))^{2}}([p, q] \times)^{2},
$$

or equivalently,

$$
\begin{aligned}
\mathcal{R} p & =q \\
\mathcal{R}[p, q] & =[p, q] .
\end{aligned}
$$

\footnotetext{
${ }^{1}$ Note the slight abuse of notation: by $\ddot{q}$ we here mean the time derivative of the components of $\dot{q}$ expressed with respect to the basis $B_{q}$. More formally, adopting the notation in (Boothby 1975), we have $\ddot{q}=\frac{D}{d t} \dot{q}$.
} 
Define the angular velocity $\omega \in \mathbb{R}^{3}$ such that $\dot{\mathcal{R}}=\mathcal{R}(\omega \times)$; let $\omega_{p} \triangleq\langle\omega, p\rangle$. We start by extending Lemma 1 on the time derivative of $\operatorname{dist}(\mathrm{p}(\mathrm{t}), \mathrm{q}(\mathrm{t}))$ to the case $q=q(t)$. By differentiating first with respect to $p$ and then with respect to $q$, we have

$$
\frac{d}{d t} \operatorname{dist}(\mathrm{p}, \mathrm{q})=-\left\langle\dot{\mathrm{p}}, \mathrm{Y}_{\mathrm{p}}^{\mathrm{q}}\right\rangle_{\mathrm{T}_{\mathrm{p}} \mathbb{S}^{2}}-\left\langle\dot{\mathrm{q}}, \mathrm{Y}_{\mathrm{q}}^{\mathrm{p}}\right\rangle_{\mathrm{T}_{\mathrm{q}} \mathbb{S}^{2}}
$$

Since equations (2.8) and (2.9) imply $\mathcal{R} Y_{p}^{q}=-Y_{q}^{p}$, we can simplify the previous expression to

$$
\frac{d}{d t} \operatorname{dist}(\mathrm{p}, \mathrm{q})=-\left\langle\dot{\mathrm{p}}-\mathcal{R}^{\mathrm{T}} \dot{\mathrm{q}}, \mathrm{Y}_{\mathrm{p}}^{\mathrm{q}}\right\rangle_{\mathrm{T}_{\mathrm{p}} \mathbb{S}^{2}}
$$

\section{Theorem 5 (Tracking of second order systems)}

Consider the system in equation (2.4). Given a positive $k_{p}$ and positive definite $K_{d}$, the control law

$$
u=\mathcal{R} \ddot{q}+k_{p} \operatorname{dist}(\mathrm{p}, \mathrm{q}) \mathrm{Y}_{\mathrm{p}}^{\mathrm{q}}-\mathrm{K}_{\mathrm{d}}(\dot{\mathrm{p}}-\mathcal{R} \dot{\mathrm{q}})+\omega_{\mathrm{p}}[\dot{\mathrm{p}}, \mathrm{p}]
$$

exponentially stabilizes $\{\operatorname{dist}(\mathrm{p}(\mathrm{t}), \mathrm{q}(\mathrm{t})), \dot{\mathrm{p}}-\mathcal{R} \dot{\mathrm{q}}\}$ to zero from any initial condition $p(0) \neq-q(0)$ and for all $k_{p}, \dot{p}(0), \dot{q}(0)$ such that

$$
k_{p}>\frac{\left\|\dot{p}(0)-\mathcal{R}(0)^{T} \dot{q}(0)\right\|^{2}}{\pi^{2}-\operatorname{dist}(\mathrm{p}(0), \mathrm{q}(0))^{2}}
$$

Additionally the scalar $\omega_{p}$ can be computed from

$$
\omega_{p}=\left\langle\tan \left(\frac{1}{2} \operatorname{dist}(\mathrm{p}, \mathrm{q})\right) \mathrm{Y}_{\mathrm{p}}^{\mathrm{q}}-\mathrm{p}, \quad[\dot{\mathrm{p}}, \mathrm{q}]+[\mathrm{p}, \dot{\mathrm{q}}]\right\rangle
$$

We refer to Appendix A for the proof.

\section{Remark 6 (Interpretation)}

Note the intuitive interpretations of all the terms present in the control law: plant inversion, proportional action, derivative action and an additional term due to the sphere curvature. If $\dot{q}=\ddot{q}=0$ this control law does not reduce to the simple PD controller shown previously. The reson being that $\omega_{p} \neq 0$ and we have an additional extra term. Of course, despite this difference, the feedback law (2.11) ensures convergence also for the regulation problem. 


\section{Spacecraft models with two control torques: projection onto the sphere}

In this section we review kinematic and dynamic models of a spacecraft actuated by two momentum wheels. We employ the following standard assumptions: the control torques are applied along the principal axes of the spacecraft and the body frame is along these principal axes, so that $J=\operatorname{diag}\left(J_{1}, J_{2}, J_{3}\right)$. Let $R \in S O(3)$ be a rotation matrix which describes the state of the system. It follows that

$$
\dot{R}=R(\omega \times)
$$

where $\omega$ is the angular velocity expressed in the body frame and the operator $\times$ is defined such that $(\omega \times) v=\omega \times x$ for all $x \in \mathbb{R}^{3}$. Following (Marsden 1992), we neglect the dynamics of the actuators and we start with a kinematic analysis. From conservation of angular momentum it follows that

$$
J \omega=R^{T} m_{0}+e_{1} v_{1}+e_{2} v_{2},
$$

where $m_{0}$ is the total constant angular momentum (vector), $e_{1}=[1,0,0]^{T}$, $e_{2}=[0,1,0]^{T}$ and the $v_{i}$ are the velocities of the wheels (scaled by the moment of inertia of the wheels about their own rotation axes). Combining (3.1) and (3.2) we have the kinematic model

$$
\dot{R}=R\left[J^{-1}\left(R^{T} m_{0}+e_{1} v_{1}+e_{2} v_{2}\right)\right] \times .
$$

A dynamic analysis (Crouch 1984) leads instead to the standard second order model

$$
\left\{\begin{aligned}
\dot{R} & =R(\omega \times), \\
J \dot{\omega} & =\left[R^{T} m_{0}, \omega\right]+e_{1} \tau_{1}+e_{2} \tau_{2},
\end{aligned}\right.
$$

where the $\tau_{i}, i=1,2$ are the torques applied to the wheels (scaled by the momentum of inertia of the wheels about their own rotation axes).

\section{Remark 7 (Gas jet actuators)}

Note that this model also applies to the case of gas jet actuators by replacing the internal drift $\left[R^{T} m_{0}, \omega\right]$ with the term $[J \omega, \omega]$ (Euler equations). See (Crouch 1984) for details.

The reduced control problem for the spacecraft models in equations (3.3) and (3.4) consists in the design of a feedback control law that stabilizes 
the state $R \in S O(3)$ up to a rotation about the unactuated principal axis $e_{1} \times e_{2}=[0,0,1]^{T}=: e_{0}$. To simplify the formulation of the problem, define the projection maps $\pi_{i}: S O(3) \rightarrow \mathbb{S}^{2}$ as $\pi_{i}(R):=R e_{i}$ (this is the same projection operator introduced in (Walsh and Sastry 1995)). Stabilizing $R$ up to a rotation about $e_{0}$ is equivalent to stabilizing the direction of the axis $R e_{0}$ and discarding the residual drift about this direction. Thus we can restate our control problem in terms of the point $\pi_{0} \in \mathbb{S}^{2}$.

\section{Problem 3 (Reduced Attitude Stabilization)}

Given the models in equations (3.3) and (3.4), find a feedback control law such as to asymptotically steer the reduced state $\pi_{0} \in \mathbb{S}^{2}$ to a fixed point $q \in \mathbb{S}^{2}$.

We now derive the reduced dynamic system corresponding to the state $\pi_{0}$. Projecting equation (3.1):

$$
\dot{\pi}_{0}=\dot{R} e_{0}=R\left(\left[\omega, e_{0}\right]\right)=-\pi_{2} \omega_{1}+\pi_{1} \omega_{2}=\left[\begin{array}{cc}
0 & 1 \\
-1 & 0
\end{array}\right]\left[\begin{array}{l}
\omega_{1} \\
\omega_{2}
\end{array}\right],
$$

with respect to the orthonormal basis $\left\{\pi_{1}, \pi_{2}\right\}$. By discarding the variable $\omega_{3}$ we write our kinematic model as

$$
\dot{\pi}_{0}=\left[\begin{array}{cc}
0 & J_{2}^{-1} \\
-J_{1}^{-1} & 0
\end{array}\right]\left\{\begin{array}{l}
\left\langle m_{0}, \pi_{1}\right\rangle \\
\left\langle m_{0}, \pi_{2}\right\rangle
\end{array}+\left[\begin{array}{l}
v_{1} \\
v_{2}
\end{array}\right]\right\}
$$

and our dynamic model as

$$
\left\{\begin{aligned}
\dot{\pi}_{0} & =\left[\begin{array}{cc}
0 & 1 \\
-1 & 0
\end{array}\right]\left[\begin{array}{l}
\omega_{1} \\
\omega_{2}
\end{array}\right] \\
{\left[\begin{array}{l}
J_{1} \dot{\omega}_{1} \\
J_{2} \dot{\omega}_{2}
\end{array}\right] } & =\left[\begin{array}{l}
\left\langle m_{0},\left[g \omega, \pi_{1}\right]\right\rangle \\
\left\langle m_{0},\left[g \omega, \pi_{2}\right]\right\rangle
\end{array}\right]+\left[\begin{array}{l}
\tau_{1} \\
\tau_{2}
\end{array}\right] .
\end{aligned}\right.
$$

Finally, note that the same reduction procedure applies to the gas jet actuators case. The dynamic equation in system (3.6) changes to

$$
\left[\begin{array}{l}
J_{1} \dot{\omega}_{1} \\
J_{2} \dot{\omega}_{2}
\end{array}\right]=\left[\begin{array}{l}
\left(J_{2}-J_{3}\right) \omega_{2} \omega_{3} \\
\left(J_{3}-J_{1}\right) \omega_{1} \omega_{3}
\end{array}\right]+\left[\begin{array}{l}
\tau_{1} \\
\tau_{2}
\end{array}\right]
$$

For the sake of completeness, we report here the expressions of the residual drift of the third component of the angular velocity:

$$
J_{3} \dot{\omega}_{3}=\left\{\begin{array}{cc}
\left\langle m_{0},\left[g \omega, \pi_{0}\right]\right\rangle & \text { momentum wheels } \\
\left(J_{1}-J_{2}\right) \omega_{1} \omega_{2} & \text { gas jet }
\end{array} .\right.
$$


Regardless of the kind of actuator, as $\omega_{1}, \omega_{2} \rightarrow 0$ the third component $\omega_{3}$ becomes constant. In particular, for the momentum wheels case, equation (3.2) projected along $e_{3}$ gives:

$$
\begin{aligned}
\omega_{3} & =J_{3}^{-1}\left\langle R^{T} m_{0}, e_{3}\right\rangle=J_{3}^{-1}\left\langle m_{0}, \pi_{0}\right\rangle \\
& =J_{3}^{-1}\left\langle m_{0}, q\right\rangle .
\end{aligned}
$$

where the last equality holds once the state has converged at its equilibrium point: $\pi_{0}=q$. In general we can conclude that $\omega_{3}$ becomes a constant of motion and that the trajectories of the spacecraft become rotations about the axis $e_{0}$.

\section{Explicit form of control laws for the spacecraft models}

The stability results obtained in Section 2 are here applied to the models introduced in Section 3. The spacecraft models are indeed fully controllable and, except for the presence of internal dynamics, satisfy the hypothesis of Theorem 2 (Regulation of first order systems) and Theorem 3 (Regulation of second order systems).

Subsection 4.1 deals briefly with the kinematic model. Assuming perfect knowledge of the plant's parameters, the first proposed control law relies on a feedforward term which exactly compensates for the dynamics of the system. The main drawback of this cancellation strategy is that various external disturbances may actually affect the rate of change of the plant's parameter $m_{0}$. These external disturbances include gravity gradients, solar radiation pressure, and Earth's magnetic field (see, for example, (Slafer and Seidenstucker 1991)). Therefore, since the dependence of the internal dynamics is linear on $m_{0}$, we propose an indirect adaptive control scheme; for details on the standard procedure see (Sastry and Bodson 1989).

Subsection 4.2 deals in full detail with the dynamic model. We give a complete solution to the reduced (spin-axis) stabilization problem through three different strategies: model independent control law (PD without feedforward term), model dependent control law (PD plus exact feedforward cancellation) and indirect adaptive control law (PD plus feedforward and adaptation law). The set of stability results that our laws achieve is very similar to what is usually obtained in the robotics literature (Wen and KreutzDelgado 1991) for passive mechanical systems: Lyapunov stability for the model independent law, exponential convergence in the case of exact feedfor- 
ward cancellation, and asymptotic stability for the indirect adaptive control scheme. A complete discussion on the proposed control laws is included.

\subsection{Proportional control laws for the kinematic model}

\section{Theorem 8 (Regulation of kinematic model)}

Consider the kinematic model in equation (3.5) and let $q \in \mathbb{S}^{2}$ be the desired goal. Given the positive definite gain $K_{p}$, the control law

$$
v=\operatorname{dist}\left(\pi_{0}, \mathrm{q}\right)\left[\begin{array}{cc}
0 & -J_{1} \\
J_{2} & 0
\end{array}\right] \mathrm{K}_{\mathrm{p}}\left[\begin{array}{c}
\left\langle Y_{\pi_{0}}^{q}, \pi_{1}\right\rangle \\
\left\langle Y_{\pi_{0}}^{q}, \pi_{2}\right\rangle
\end{array}\right]-\left[\begin{array}{l}
\left\langle m_{0}, \pi_{1}\right\rangle \\
\left\langle m_{0}, \pi_{2}\right\rangle
\end{array}\right]
$$

exponentially stabilizes the state $\pi_{0}(t)$ at the goal $q$ from any initial condition $\pi_{0}(0) \neq-q$ and with time-constant at least $1 / \lambda_{\min }\left(K_{p}\right)$.

Proof. Simply note that the trajectories of the closed-loop system satisfy

$$
\dot{\pi}_{0}=\operatorname{dist}\left(\pi_{0}, \mathrm{q}\right) \mathrm{K}_{\mathrm{p}}\left[\begin{array}{l}
\left\langle Y_{\pi_{0}}^{q}, \pi_{1}\right\rangle \\
\left\langle Y_{\pi_{0}}^{q}, \pi_{2}\right\rangle
\end{array}\right]
$$

Theorem 2 applies.

\section{Remark 9 (Inertia matrix unknown)}

If $\left\{J_{1}, J_{2}\right\}$ are not known (while the angular momentum $m_{0}$ is), then the control law:

$$
u=-\left[\begin{array}{c}
\left\langle\pi_{1}, m_{0}\right\rangle \\
\left\langle\pi_{2}, m_{0}\right\rangle
\end{array}\right]+\operatorname{dist}\left(\pi_{0}, \mathrm{q}\right)\left[\begin{array}{cc}
0 & -1 \\
1 & 0
\end{array}\right] \mathrm{K}_{\mathrm{p}}\left[\begin{array}{c}
\left\langle Y_{\pi_{0}}^{q}, \pi_{1}\right\rangle \\
\left\langle Y_{\pi_{0}}^{q}, \pi_{2}\right\rangle
\end{array}\right],
$$

still ensures exponential stability of the equilibrium point $q$ with timeconstant at least $1 / \lambda_{\min }\left(\operatorname{diag}\left(J_{2}^{-1}, J_{1}^{-1}\right) K_{p}\right)$. Given $W=\operatorname{dist}\left(\pi_{0}, \mathrm{q}\right)^{2} / 2$, we have:

$$
\begin{aligned}
\dot{W} & =-\operatorname{dist}\left(\pi_{0}, \mathrm{q}\right)^{2}\left\langle\left[\begin{array}{cc}
J_{2}^{-1} & 0 \\
0 & J_{1}^{1} \\
K_{p}
\end{array}\right]\left[\begin{array}{c}
\left\langle Y_{\pi_{0}}^{q}, \pi_{1}\right\rangle \\
\left\langle Y_{\pi_{0}}^{q}, \pi_{2}\right\rangle
\end{array}\right],\left[\begin{array}{c}
\left\langle Y_{\pi_{0}}^{q}, \pi_{1}\right\rangle \\
\left\langle Y_{\pi_{0}}^{q}, \pi_{2}\right\rangle
\end{array}\right]\right\rangle \\
& \leq-2 \lambda_{\min }\left(\operatorname{diag}\left(J_{2}^{-1}, J_{1}^{-1}\right) K_{p}\right) W .
\end{aligned}
$$

Assume now that the inertia matrix is known and that only have an estimate of the angular momentum $m_{0}$ is available. Then, we can design a classical indirect adaptive control scheme: 


\section{Theorem 10 (Adaptive regulation of kinematic model)}

Consider system in equation (3.5) and let $\widehat{m}_{0}$ be the current estimate of the unknown parameter $m_{0}$. Let the control gain $K_{p}$ and the adaptation gain $\Gamma$ be symmetric, positive definite matrices. Then the control law

$$
v=\operatorname{dist}\left(\pi_{0}, \mathrm{q}\right)\left[\begin{array}{cc}
0 & -J_{1} \\
J_{2} & 0
\end{array}\right] \mathrm{K}_{\mathrm{p}}\left[\begin{array}{c}
\left\langle Y_{\pi_{0}}^{q}, \pi_{1}\right\rangle \\
\left\langle Y_{\pi_{0}}^{q}, \pi_{2}\right\rangle
\end{array}\right]-\left[\begin{array}{c}
\left\langle\pi_{1}, \widehat{m}_{0}\right\rangle \\
\left\langle\pi_{2}, \widehat{m}_{0}\right\rangle
\end{array}\right]
$$

(based on the certainty equivalence principle) and the update law

$$
\frac{d}{d t} \widehat{m}_{0}=-\operatorname{dist}\left(\pi_{0}, \mathrm{q}\right) \Gamma \mathrm{RJ}^{-1} \mathrm{R}^{\mathrm{T}} \operatorname{vers}\left[\pi_{0}, \mathrm{q}\right],
$$

locally, asymptotically stabilize the state $\pi_{0}(t)$ at the goal $q \in \mathbb{S}^{2}$ and make $m_{0}-\widehat{m}_{0}$ go to a constant.

Appendix B contains the detailed proof; here instead let us comment on the word "locally". Under that we actually mean the following sufficient upper bound on the initial conditions:

$$
\operatorname{dist}\left(\pi_{0}(0), q\right)^{2}+\left\|m_{0}-\widehat{m}_{0}(0)\right\|_{\Gamma^{-1}}^{2}<\pi^{2} .
$$

Note that decreasing the adaptation gain $\Gamma$ (in this matrix case, decreasing the eigenvalues of $\Gamma$ ) makes this bound as loose as desired. If the condition is verified, then $\operatorname{dist}\left(\pi_{0}, \mathrm{q}\right)<\pi$ for all $t$ (proof in Appendix B) and the singularity of the control law is never reached.

\subsection{Proportional plus derivative control laws for the dynamic model}

All the stability results in this section rely on a skewed mechanical metric on $\mathbb{S}^{2}$ to design the Lyapunov function.

The simplest control law we propose is a PD controller without feedforward cancellation; since no knowledge of the model is required we call this control "model independent". The stability proof is based on the fact that the kinetic energy of the full spacecraft is constant in absence of control inputs.

\section{Theorem 11 (Model independent regulation)}

Consider the dynamic model in equation (3.6). Given the positive $k_{p}$ and the positive definite $K_{d}$, the control law

$$
\tau=k_{p} \operatorname{dist}\left(\pi_{0}, \mathrm{q}\right)\left[\begin{array}{c}
-\left\langle Y_{\pi_{0}}^{q}, \pi_{2}\right\rangle \\
\left\langle Y_{\pi_{0}}^{q}, \pi_{1}\right\rangle
\end{array}\right]-\mathrm{K}_{\mathrm{d}}\left[\begin{array}{l}
\omega_{1} \\
\omega_{2}
\end{array}\right],
$$


renders the equilibrium point $\left(q,\left[\begin{array}{ll}0 & 0\end{array}\right]^{T}\right)$ Lyapunov stable (not necesseraly asymptotically stable) from any initial condition $\pi_{0}(0) \neq-q$ and for all $k_{p}$ and $\omega$ such that

$$
k_{p}>\frac{\langle\omega, J \omega\rangle}{\pi^{2}-\operatorname{dist}\left(\pi_{0}(0), q\right)^{2}},
$$

Additionally the $\operatorname{distance} \operatorname{dist}\left(\pi_{0}(\mathrm{t}), \mathrm{q}\right)$ converges to a constant $\bar{d} \leq\left\|m_{0}\right\|^{2} /\left(2 J_{3} k_{p}\right)$.

Proof. Consider as state of the system $\left(\pi_{0}, \omega\right) \in \mathbb{S}^{2} \times \mathbb{R}^{3}$ and let

$$
W \triangleq \frac{k_{p}}{2} \operatorname{dist}\left(\pi_{0}, q\right)^{2}+\langle\omega, \mathrm{J} \omega\rangle
$$

be our candidate Lyapunov function. Recall that the full state dynamic equation is

$$
J \dot{\omega}=\left[R^{T} m_{0}, \omega\right]+\left[\begin{array}{c}
\tau_{1} \\
\tau_{2} \\
0
\end{array}\right]
$$

and take the time derivative of $W$ :

$$
\begin{aligned}
\dot{W} & =-k_{p} \operatorname{dist}\left(\pi_{0}, \mathrm{q}\right)\left\langle\left[\begin{array}{c}
\omega_{1} \\
\omega_{2}
\end{array}\right],\left[\begin{array}{c}
-\left\langle Y_{\pi_{0}}^{q}, \pi_{2}\right\rangle \\
\left\langle Y_{\pi_{0}}^{q}, \pi_{1}\right\rangle
\end{array}\right]\right\rangle+\left\langle\left[\begin{array}{l}
\omega_{1} \\
\omega_{2}
\end{array}\right],\left[\begin{array}{l}
\tau_{1} \\
\tau_{2}
\end{array}\right]\right\rangle+\left\langle\omega,\left[\mathrm{g}^{\mathrm{T}} \mathrm{m}_{0}, \omega\right]\right\rangle \\
& =-k_{p} \operatorname{dist}\left(\pi_{0}, \mathrm{q}\right)\left\langle\left[\begin{array}{c}
\omega_{1} \\
\omega_{2}
\end{array}\right],\left[\begin{array}{c}
-\left\langle Y_{\pi_{0}}^{q}, \pi_{2}\right\rangle \\
\left\langle Y_{\pi_{0}}^{q}, \pi_{1}\right\rangle
\end{array}\right]\right\rangle+\left\langle\left[\begin{array}{l}
\omega_{1} \\
\omega_{2}
\end{array}\right],\left[\begin{array}{l}
\tau_{1} \\
\tau_{2}
\end{array}\right]\right\rangle,
\end{aligned}
$$

since the last term is null (the drift does not affect the kinetic energy). As in the proofs of Section 2, the proportional term cancels the derivative of the distance-square term:

$$
\begin{aligned}
\dot{W}= & -k_{p} \operatorname{dist}\left(\pi_{0}, \mathrm{q}\right)\left\langle\left[\begin{array}{l}
\omega_{1} \\
\omega_{2}
\end{array}\right],\left[\begin{array}{c}
-\left\langle Y_{\pi_{0}}^{q}, \pi_{2}\right\rangle \\
\left\langle Y_{\pi_{0}}^{q}, \pi_{1}\right\rangle
\end{array}\right]\right\rangle \\
& +\left\langle\left[\begin{array}{l}
\omega_{1} \\
\omega_{2}
\end{array}\right], k_{p} \operatorname{dist}\left(\pi_{0}, \mathrm{q}\right)\left[\begin{array}{c}
-\left\langle Y_{\pi_{0}}^{q}, \pi_{2}\right\rangle \\
\left\langle Y_{\pi_{0}}^{q}, \pi_{1}\right\rangle
\end{array}\right]-\mathrm{K}_{\mathrm{d}}\left[\begin{array}{l}
\omega_{1} \\
\omega_{2}
\end{array}\right]\right\rangle \\
= & -\left\|\left[\begin{array}{l}
\omega_{1} \\
\omega_{2}
\end{array}\right]\right\|_{K_{d}}^{2} .
\end{aligned}
$$

Since the Lyapunov function $W$ is decreasing, the bound in equation (4.1) ensures the smoothness of the control law. Lyapunov stability is therefore proved. Additionally, by Lasalle's principle it follows that

$$
\lim _{t \rightarrow \infty} \omega_{1}(t)=\lim _{t \rightarrow \infty} \omega_{2}(t)=0
$$


so that even $\frac{d}{d t} \pi_{0}$ goes to zero and the error $\operatorname{dist}\left(\pi_{0}, \mathrm{q}\right)$ to a constant. Indeed in $\Omega$ we have

$$
0=\left[\begin{array}{l}
\left\langle\left[g^{T} m_{0}, \omega\right], e_{1}\right\rangle \\
\left\langle\left[g^{T} m_{0}, \omega\right], e_{2}\right\rangle
\end{array}\right]+k_{p} \operatorname{dist}\left(\pi_{0}, \mathrm{q}\right)\left[\begin{array}{c}
-\left\langle Y_{\pi_{0}}^{q}, \pi_{2}\right\rangle \\
\left\langle Y_{\pi_{0}}^{q}, \pi_{1}\right\rangle
\end{array}\right]
$$

which leads to the asymptotic bound

$$
\operatorname{dist}\left(\pi_{0}, \mathrm{q}\right) \leq \frac{\mathrm{J}_{3}^{-1}}{\mathrm{k}_{\mathrm{p}}}\left|\left\langle\mathrm{m}_{0}, \pi_{0}\right\rangle\right| \cdot\left\|\left[\mathrm{m}_{0}, \pi_{0}\right]\right\| \leq \frac{\left\|\mathrm{m}_{0}\right\|^{2}}{2 \mathrm{~J}_{3} \mathrm{k}_{\mathrm{p}}} .
$$

Note that increasing the gain $k_{p}$ makes this bound as tight as desired.

The second proposed control law assumes exact cancellation between internal dynamics and feedforward control so as to satisfy the hypothesis of Theorem 3. Since exact knowledge of the model is required, we call this control law "model dependent".

Theorem 12 (Model dependent regulation)

Consider the system in equation (3.6). Given the positive definite gains $K_{p}$ and $K_{d}$, the control law

$$
\tau=\operatorname{dist}\left(\pi_{0}, \mathrm{q}\right) \mathrm{K}_{\mathrm{p}}\left[\begin{array}{c}
-\left\langle Y_{\pi_{0}}^{q}, \pi_{2}\right\rangle \\
\left\langle Y_{\pi_{0}}^{q}, \pi_{1}\right\rangle
\end{array}\right]-\mathrm{K}_{\mathrm{d}}\left[\begin{array}{l}
\omega_{1} \\
\omega_{2}
\end{array}\right]-\left[\begin{array}{l}
\left\langle m_{0},\left[g \omega, \pi_{1}\right]\right\rangle \\
\left\langle m_{0},\left[g \omega, \pi_{2}\right]\right\rangle
\end{array}\right]
$$

exponentially stabilizes the state $\pi_{0}(t)$ at the goal $q$ from any initial condition $\pi_{0}(0) \neq-q$ and for all $K_{p}$ and $\omega$ such that

$$
\lambda_{\min }\left(K_{p}\right)>\frac{J_{1} \omega_{1}^{2}+J_{2} \omega_{2}^{2}}{\pi^{2}-\operatorname{dist}\left(\pi_{0}(0), q\right)^{2}} .
$$

Proof. The proof is the same as Theorem 3, with the only difference that a skewed mechanical metric is used in designing the candidate Lyapunov function. Simply define

$$
\begin{aligned}
W \triangleq & \frac{1}{2} \operatorname{dist}\left(\pi_{0}, \mathrm{q}\right)^{2}+\frac{1}{2}\left\langle\left[\begin{array}{l}
\omega_{1} \\
\omega_{2}
\end{array}\right], \mathrm{K}_{\mathrm{p}}^{-1}\left[\begin{array}{cc}
J_{1} & 0 \\
0 & J_{2}
\end{array}\right]\left[\begin{array}{l}
\omega_{1} \\
\omega_{2}
\end{array}\right]\right\rangle \\
& -\epsilon \operatorname{dist}\left(\pi_{0}, \mathrm{q}\right)\left\langle\left[\begin{array}{l}
\omega_{1} \\
\omega_{2}
\end{array}\right],\left[\begin{array}{c}
-\left\langle Y_{\pi_{0}}^{q}, \pi_{2}\right\rangle \\
\left\langle Y_{\pi_{0}}^{q}, \pi_{1}\right\rangle
\end{array}\right]\right\rangle,
\end{aligned}
$$

and then follow the steps of the proof of Theorem 3 (including Appendix A). As usual, condition (4.2) is imposed to confine the closed-loop trajectories away from the singularity. 
As already explained, exact cancellation of internal drift is not a robust procedure, in that the total angular momentum $m_{0}$ might either be unknown or change slowly in time (see discussion at the beginning of this section). Therefore, as for the kinematic model, we design an indirect adaptive control scheme:

\section{Theorem 13}

(Indirect Adaptive Regulation) Consider the system in equation (3.6). Let $\widehat{m}_{0}$ be the current estimate of $m_{0}$, let the control gain $K_{p}, K_{d}$ and the adaptation gain $\Gamma$ be symmetric, positive definite matrices. Then the control law

$$
\tau=\operatorname{dist}\left(\pi_{0}, \mathrm{q}\right) \mathrm{K}_{\mathrm{p}}\left[\begin{array}{c}
-\left\langle Y_{\pi_{0}}^{q}, \pi_{2}\right\rangle \\
\left\langle Y_{\pi_{0}}^{q}, \pi_{1}\right\rangle
\end{array}\right]-\mathrm{K}_{\mathrm{d}}\left[\begin{array}{l}
\omega_{1} \\
\omega_{2}
\end{array}\right]-\left[\begin{array}{c}
\left.\left\langle\widehat{m}_{0},\left[g \omega, \pi_{1}\right]\right\rangle\right] \\
\left\langle\widehat{m}_{0},\left[g \omega, \pi_{2}\right]\right\rangle
\end{array}\right],
$$

and the update law

$$
\frac{d}{d t} \widehat{m}_{0}=\Gamma g(\omega \times)\left[\begin{array}{c}
K_{p}^{-1}\left[\begin{array}{l}
\omega_{1} \\
\omega_{2}
\end{array}\right] \\
0
\end{array}\right]-\epsilon \operatorname{dist}\left(\pi_{0}, \mathrm{q}\right) \mathrm{g}(\omega \times) \mathrm{J}^{-1} \operatorname{vers}\left[\mathrm{e}_{0}, \mathrm{R}^{\mathrm{T}} \mathrm{q}\right],
$$

locally, asymptotically stabilize the equilibrium point $q \in \mathbb{S}^{2}$ for sufficiently small $\epsilon$.

Proof. For the detailed proof see Appendix B.

Let us now comment on the proposed laws:

\section{Remark 14 (Trade-off between the proposed laws)}

Each of the three proposed strategies has its own strengths and weaknesses. From an applications viewpoint, the choice of control law can be taken on the basis of meaningful parameters, such as controller complexity and stability properties versus computational load, or required a priori knowledge of the plant's parameter and of the external disturbances. For a complete discussion on this issue we refer for example to (Wen and Kreutz-Delgado 1991), where a full set of model independent, model dependent and indirect adaptive control laws is also proposed (but for the attitude stabilization problem).

\section{Remark 15 (A family of simplified control laws)}

The proportional action, as described in Section 2, is:

$$
\operatorname{dist}(\mathrm{p}, \mathrm{q}) \mathrm{Y}_{\mathrm{p}}^{\mathrm{q}} \equiv \frac{\theta}{\sin \theta}[[\mathrm{p}, \mathrm{q}], \mathrm{p}]
$$


where $\theta=\operatorname{dist}(\mathrm{p}, \mathrm{q})$. This expression immediately suggests a simplification; assuming $\sin (\theta) \approx \theta$, the model dependent control law looks like

$$
\tau=-\operatorname{drift}+K_{p}\left[\begin{array}{c}
-\left\langle q, \pi_{2}\right\rangle \\
\left\langle q, \pi_{1}\right\rangle
\end{array}\right]-K_{d}\left[\begin{array}{l}
\omega_{1} \\
\omega_{2}
\end{array}\right] .
$$

The stability properties of this sin-like control law can be verified through the following candidate Lyapunov function:

$$
W=\left(1-\cos \left(\operatorname{dist}\left(\pi_{0}, \mathrm{q}\right)\right)\right)+\left\langle\left[\begin{array}{l}
\omega_{1} \\
\omega_{2}
\end{array}\right], \mathrm{K}_{\mathrm{p}}^{-1}\left[\begin{array}{l}
\omega_{1} \\
\omega_{2}
\end{array}\right]\right\rangle,
$$

where the cosine is introduced to maintain the cancellation which occurs in the derivative of $W$. The usual steps lead to

$$
\dot{W}=-\left\|\left[\begin{array}{c}
\omega_{1} \\
\omega_{2}
\end{array}\right]\right\|_{K_{p}^{-1} K_{d}}^{2},
$$

proving the Lyapunov stability of the closed-loop trajectories of the system. Indeed, exponential stability can be easily proved, since close to the origin $\left(\operatorname{small} \operatorname{dist}\left(\pi_{0}, q\right)\right)$ new and old strategies do not differ substantially.

This simplified control law has the following two main differences with respect to the original one: first, the law is smooth on all $\mathbb{S}^{2}$ and the point $-q$ becomes an instable equilibrium point. Second, the new law has the drawback of exerting a decreasing control for an increasing distance of state and goal when the state is more distant than $\pi / 2$.

\section{Remark 16 (Gas jet actuators)}

So far we have dealt with momentum wheel actuators, but the proposed control laws also apply to the case of gas jets. The model independent control law remains unchanged:

$$
\tau=k_{p} \operatorname{dist}\left(\pi_{0}, \mathrm{q}\right)\left[\begin{array}{c}
-\left\langle Y_{\pi_{0}}^{q}, \pi_{2}\right\rangle \\
\left\langle Y_{\pi_{0}}^{q}, \pi_{1}\right\rangle
\end{array}\right]-\mathrm{K}_{\mathrm{d}}\left[\begin{array}{c}
\omega_{1} \\
\omega_{2}
\end{array}\right],
$$

and Lyapunov stability can be proved easily through the same proof of Theorem 11. In the model dependent control law, we simply compensate for the different drift:

$$
\tau=\operatorname{dist}\left(\pi_{0}, \mathrm{q}\right) \mathrm{K}_{\mathrm{p}}\left[\begin{array}{c}
-\left\langle Y_{\pi_{0}}^{q}, \pi_{2}\right\rangle \\
\left\langle Y_{\pi_{0}}^{q}, \pi_{1}\right\rangle
\end{array}\right]-\mathrm{K}_{\mathrm{d}}\left[\begin{array}{l}
\omega_{1} \\
\omega_{2}
\end{array}\right]-\left[\begin{array}{l}
a_{1} \omega_{2} \omega_{3} \\
a_{2} \omega_{1} \omega_{3}
\end{array}\right],
$$

where $a_{1}=J_{2}-J_{3}, a_{2}=J_{3}-J_{1}$. As before, condition (4.2) ensures the smoothness of the control law. 
Note that the control law in equation (4.5) relies on exact knowledge of the inertia matrix and is not robust with respect to retrieval or deployment of unknown payloads. Hence we design a locally, asymptotically adaptive control scheme based on a certainty equivalence control

$$
\tau=\operatorname{dist}\left(\pi_{0}, \mathrm{q}\right) \mathrm{K}_{\mathrm{p}}\left[\begin{array}{c}
-\left\langle Y_{\pi_{0}}^{q}, \pi_{2}\right\rangle \\
\left\langle Y_{\pi_{0}}^{q}, \pi_{1}\right\rangle
\end{array}\right]-\mathrm{K}_{\mathrm{d}}\left[\begin{array}{c}
\omega_{1} \\
\omega_{2}
\end{array}\right]-\left[\begin{array}{l}
\widehat{a}_{1} \omega_{2} \omega_{3} \\
\widehat{a}_{2} \omega_{1} \omega_{3}
\end{array}\right]
$$

coupled with the update law

$$
\frac{d}{d t}\left[\begin{array}{l}
\widehat{a}_{1} \\
\widehat{a}_{2}
\end{array}\right]=\gamma\left[\begin{array}{l}
\omega_{1} \omega_{2} \omega_{3} \\
\omega_{1} \omega_{2} \omega_{3}
\end{array}\right]
$$

for all $\gamma>0$. The stability proof is standard.

\section{Simulations}

To verify theoretical predictions and to gain insight into the stability properties, we run two sets of simulations. In the first one we implemented the the dynamic model of a spacecraft with momentum wheels and the regulation algorithms in Section 4. In the second set, we simulated a general second order systems on the sphere and we implemented the trajectory tracking law of Subsection 2.2.

\subsection{Reduced attitude regulation of the dynamic spacecraft}

The full system defined on $S O(3) \times \mathbb{R}^{3}$ is simulated:

$$
\left\{\begin{aligned}
\dot{R} & =R(\omega \times), \\
J \omega & =\left[R^{T} m_{0}, \omega\right]+\left[\begin{array}{c}
\tau_{1} \\
\tau_{2} \\
0
\end{array}\right] .
\end{aligned}\right.
$$

Note that the attitude matrix $R$ is not a suitable parametrization of $S O(3)$ for computational goals: it includes too much redundancy and we would have to project from $G L(3)$ to $S O(3)$ at each step. Instead we use the unit quaternion parametrization, which minimizes the redundancy while at the same time avoiding singularities in the representation. Let us recall that, if $\dot{R}=R(\omega \times)$, the corresponding unit quaternion $\left(q_{0}, \vec{q}\right) \in \mathbb{R}^{4}$ satisfies

$$
\frac{d}{d t}\left[\begin{array}{c}
q_{0} \\
\vec{q}
\end{array}\right]=\frac{1}{2}\left[\begin{array}{c}
\langle\omega, \vec{q}\rangle \\
q_{0} \omega+[\vec{q}, \omega]
\end{array}\right] .
$$




\begin{tabular}{|llll|}
\hline Inertia matrix & $J=\operatorname{diag}(1.0,0.63,0.87)$ \\
Angular momentum & $m_{0}=[1,1,1]^{T}$ \\
Proportional gain: scalar & $k_{p}=5$ \\
& $K_{p}=\left[\begin{array}{ll}3.0 & 0.5 \\
0.5 & 1.5\end{array}\right]$ \\
Proportional gain & $K_{d}=\left[\begin{array}{ll}3.0 & 0.3 \\
0.3 & 1.5\end{array}\right]$ \\
& & & \\
Derivative gain & $\gamma$ & $=1$ \\
Adaptation gain: scalar & $\gamma$ & \\
Adaptation gain: scalar & $\epsilon$ & $=[1,0,0]^{T}$ \\
Desired orientation & $q$ & $=0.01 \mathrm{secs}$ \\
Time step & $h$ &
\end{tabular}

Table 1: Table of parameters for simulations.

To enforce the unit norm constraint on the numerical solution of the differential equation, we adopt a projection procedure.

We implemented the model independent control law and the adaptive scheme as in Theorems 11 and 13. An explicit expression for the adaptive control law is

$$
\tau=\left[\begin{array}{l}
\left\langle\left[\omega, R^{T} \widehat{m}_{0}\right], e_{1}\right\rangle \\
\left\langle\left[\omega, R^{T} \widehat{m}_{0}\right], e_{2}\right\rangle
\end{array}\right]+\frac{\arcsin \|z\|}{\|z\|} K_{p} z-K_{d}\left[\begin{array}{l}
\omega_{1} \\
\omega_{2}
\end{array}\right]
$$

where

$$
z \triangleq\left[\begin{array}{c}
-\left\langle R^{T} q, e_{2}\right\rangle \\
\left\langle R^{T} q, e_{1}\right\rangle
\end{array}\right]
$$

and where we use $\arcsin :[-1,1] \rightarrow[-\pi / 2, \pi / 2]$ instead of arccos $:[-1,1] \rightarrow$ $[0, \pi]$ to avoid numerical singularities when $\|z\|=\sin \left(\operatorname{dist}\left(\pi_{0}, q\right)\right)$ is small. The estimate of the parameter $\widehat{m}_{0}$ follows the update law of Theorem 13 .

The numerical integrator of ODE is a $4^{\text {th }}$ order Runge Kutta, the simulations were implemented on Matlab and were run on a Sun Sparcstation. Initial conditions were $R(0)=I_{3}$ and $\omega(0)=J^{-1} R^{T} m_{0}$. The final desired orientation was $q=[1,0,0]^{T}$. The parameters of plant, controller and simulation, are listed in Table 1 . Note that

$$
\pi_{0}(0)=I_{3} e_{0}=e_{0}=\left[\begin{array}{l}
0 \\
0 \\
1
\end{array}\right], \quad q=e_{1}=\left[\begin{array}{l}
1 \\
0 \\
0
\end{array}\right],
$$


and therefore $\operatorname{dist}\left(\pi_{0}(0), q\right)=\pi / 2$. The initial kinetic energy of the spacecraft is

$$
K(0)=\langle\omega(0), J \omega(0)\rangle=\left\langle J^{-1} m_{0}, m_{0}\right\rangle=2.5,
$$

so that

$$
\lambda_{\min }\left(K_{p}\right) \geq \frac{\langle\omega(0), J \omega(0)\rangle}{\pi^{2}-\operatorname{dist}\left(\pi_{0}(0), \mathrm{q}\right)}=\frac{10}{3 \pi} \approx 1.06
$$

is a sufficient condition for the smoothness of the control law for the model independent control law.

The numerical simulations are reported in Figure 2 on the facing page (model independent control), and Figure 3 on page 24 (indirect adaptive control). In both cases we include: $\pi_{0} \in \mathbb{S}^{2} \subset \mathbb{R}^{3}$ expressed in inertial coordinates (unitless), the distance $\operatorname{dist}\left(\pi_{0}, q\right)$ and the angular velocity $\omega$. For the second simulation (adaptive control scheme) we also show the estimate of the angular momentum $\widehat{m}_{0}$. Regarding the model independent control law: As in the theoretical analysis (Theorem 11), the distance between $\pi_{0}$ and $q$ goes to a constant (second picture), which satisfies bound (4.1). Indeed the first two components of the angular velocity go to zero, while the third one becomes a constant (third picture). Regarding the adaptive control law: As in the theoretical analysis (Theorem 13), the state of the spacecraft converges (at least) asymptotically to the desired equilibrium configuration (see second picture for $\operatorname{dist}\left(\pi_{0}, q\right)$ and third picture for $\omega_{1}$ and $\omega_{2}$ ), while the estimation error goes to a steady state generically different from zero (see fourth picture). 

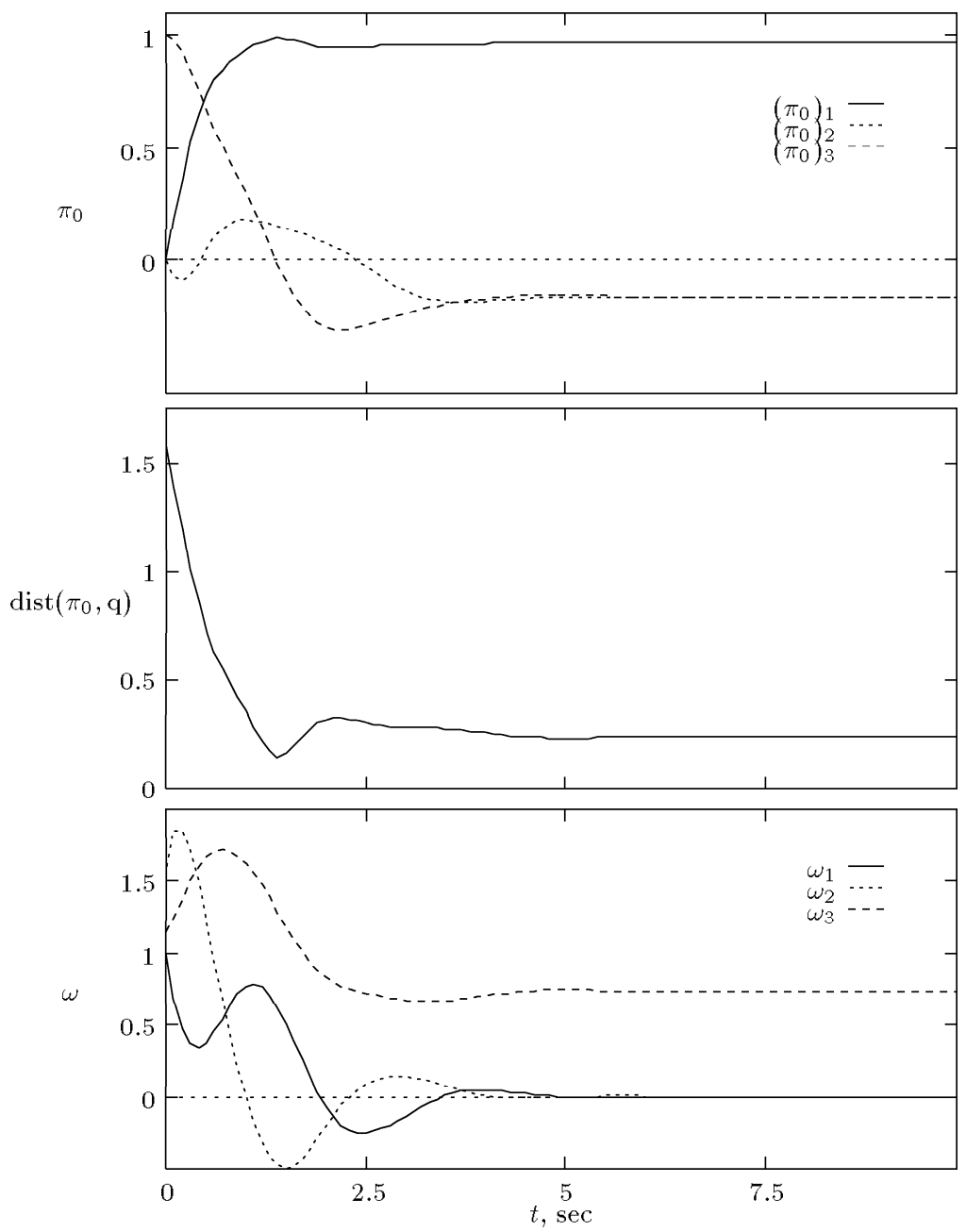

Figure 2: Simulation for the model independent control law (as in Theorem 11). 

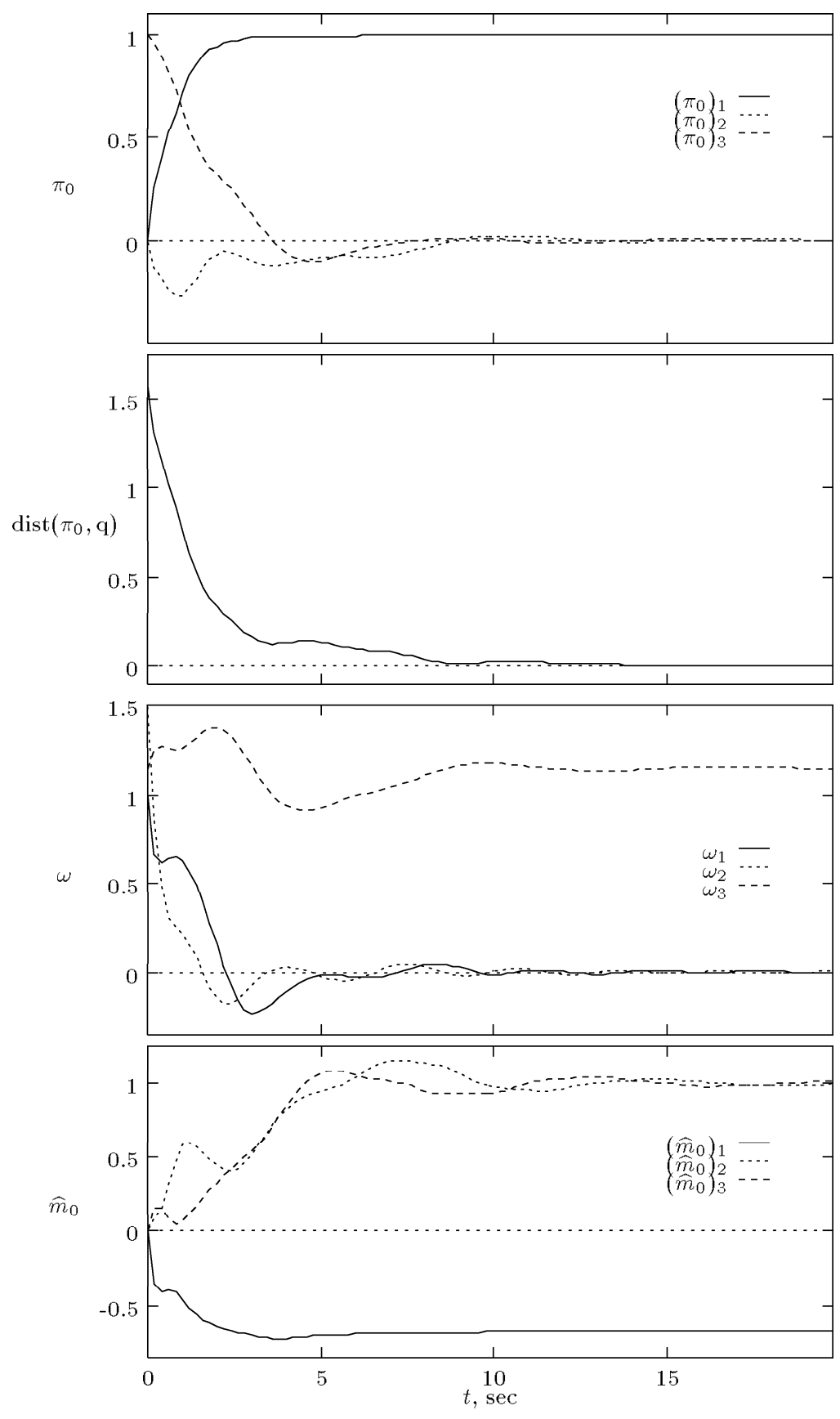

Figure 3: Simulation for indirect adaptive control scheme (as in Theorem 13). 


\subsection{Trajectory tracking on the sphere}

We simulated the dynamic system in equation (2.4) and the PD control law in Theorem 5. The most instructive reason for performing such a simulation is to test the importance of the weird additional term $\omega_{p}$ in the control law. Indeed recall that the control law (2.11) is

$$
u=\mathcal{R} \ddot{\mathrm{q}}+k_{p} \operatorname{dist}(\mathrm{p}, \mathrm{q}) \mathrm{Y}_{\mathrm{p}}^{\mathrm{q}}-\mathrm{K}_{\mathrm{d}}(\dot{\mathrm{p}}-\mathcal{R} \dot{\mathrm{q}})+\omega_{\mathrm{p}}[\dot{\mathrm{p}}, \mathrm{p}],
$$

where the feedforward term $\mathcal{R} \ddot{q}$ performs a plant inversion, $k_{p}$ and $K_{d}$ correpond to proportional and derivative action and the additional (with respect to the standard $\mathbb{R}^{n}$ case) term $\omega_{p}[\dot{p}, p]$ represents some kind of a "curvature" correction term.

Surprisingly enough, despite the technical need for such a correction term in the stability proof, the control law performs equally well even without it. As a matter of fact in our first simulation (see Figure 4 on the following page) we show the experimental data obtained without the presence of such term. We don't report the full control law, since the performances are approximately equal: exponential tracking is achieved as foreseen in the theoretical analysis.

In the second simulation (see Figure 5 on page 27) we implement an even more simplified control law, where even the feedforward term vanishes. This corresponds to the concrete case in which the acceleration of the desired signal $\ddot{q}$ is unknown. The control law shows a still satisfying performance and actually tracks the signal achieving a bounded error response.

Just a few words about the details of the simulations: in both cases the desired goal $q(t) \in \mathbb{S}^{2}$ behaves under the effects of a gravitational-like acceleration field $\ddot{q}$ and of a small damping. The initial conditions can be easily observed from the various plots; the controller's gains are $k_{p}=2$ and $k_{d}=1$. 

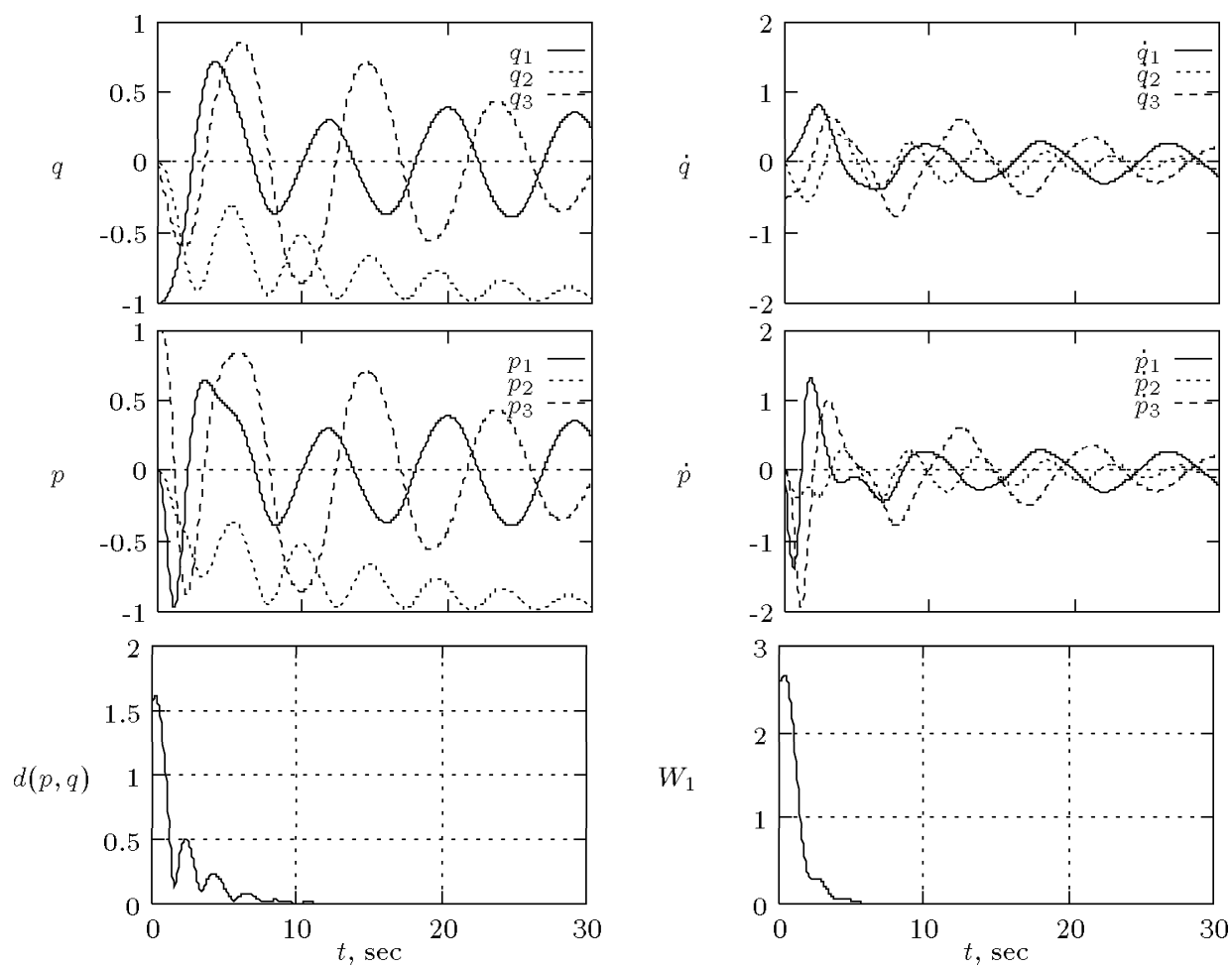

Figure 4: Simulation for the trajectory tracking law (as in Theorem 5): PD controller with feedforward term. We report: the state $p(t) \in \mathbb{S}^{2}$, its velocity $\dot{p} \in T_{p} \mathbb{S}^{2}$; the desired position $q(t) \in \mathbb{S}^{2}$, its velocity $\dot{q} \in T_{q} \mathbb{S}^{2}$; the distance between state and goal $\operatorname{dist}(\mathrm{p}(\mathrm{t}), \mathrm{q}(\mathrm{t}))$ and the Lyapunov function $W_{1}(t)$ as defined in equation (A.5). 

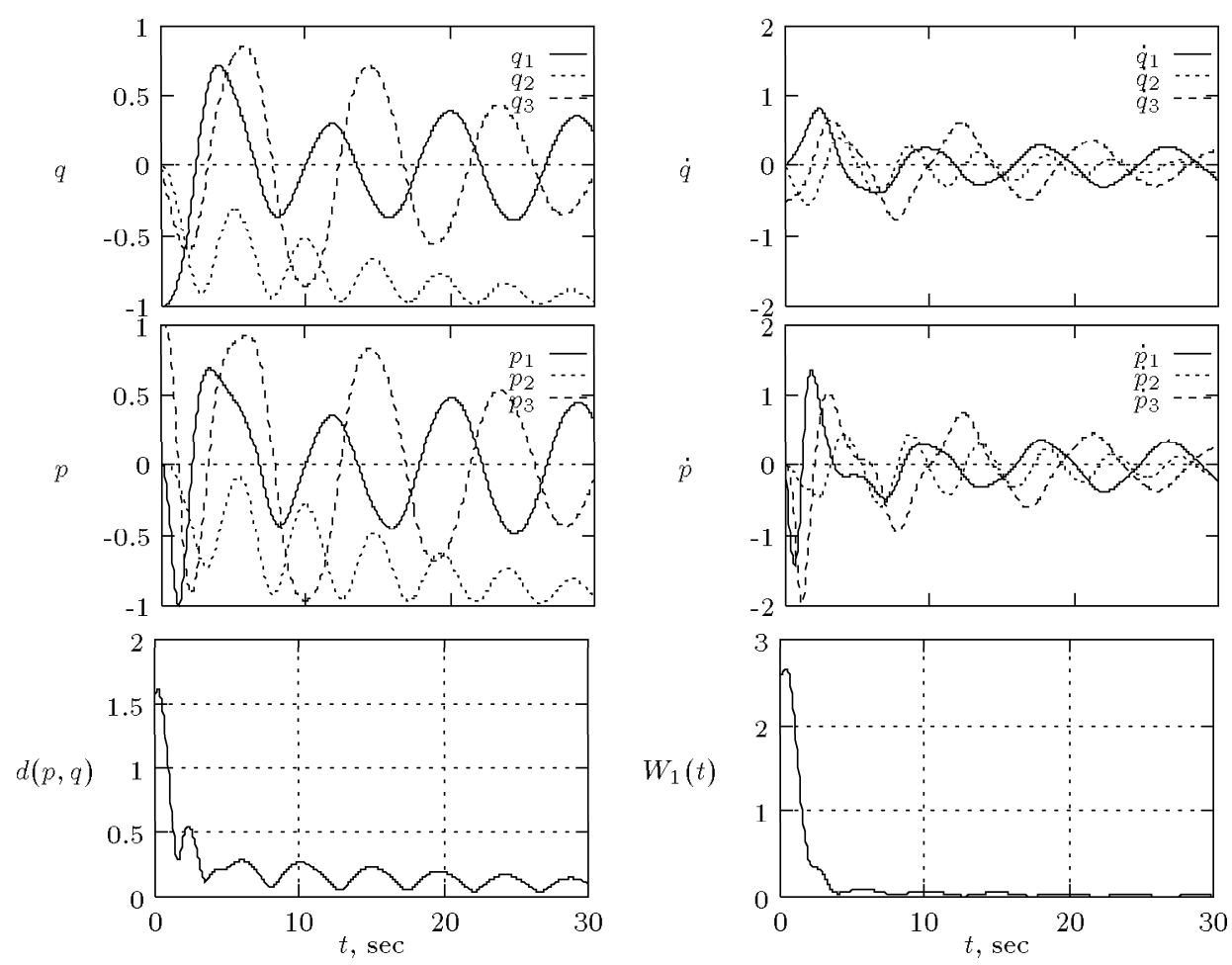

Figure 5: Simulation for the trajectory tracking law (as in Theorem 5): simple PD control law without the feedforward term. As before we report: the state $p(t) \in \mathbb{S}^{2}$, its velocity $\dot{p} \in T_{p} \mathbb{S}^{2}$; the desired position $q(t) \in \mathbb{S}^{2}$, its velocity $\dot{q} \in T_{q} \mathbb{S}^{2}$; the distance between state and goal $\operatorname{dist}(\mathrm{p}(\mathrm{t}), \mathrm{q}(\mathrm{t}))$ and the Lyapunov function $W_{1}(t)$ as defined in equation (A.5). 


\section{Conclusions}

In this paper we have dealt with fully actuated control systems defined on the sphere $\mathbb{S}^{2}$ and in such setting we have proposed a novel approach to regulation and trajectory tracking problems. Our results have then applied to a reduced attitude stabilization problem (spin-axis stabilization). We have designed a comprehensive set of control laws, which differ in stability properties, computational complexity and required model knowledge. By and large, our (differential) geometric approach has lead to a family of simple, versatile and robust control laws.

The work proposed here can be seen as a development of previous investigations on the correct Lyapunov function's design (Koditschek 1989). We rely on the Riemannian notion of distance to achieve a simple and successful solution to global problems such as trajectory tracking. The simplicity and efficacy of this approach can then apply to more general Riemannian manifolds. Indeed, control systems defined on Lie groups belong to this class and provide a very instructive example. Here the topological properties of the group, such as the compactness, influence its metric structure and only in certain cases our approach applies straightforwardly; for an introduction see (Bullo and Murray 1994).

\section{Acknowledgments}

This research was supported in part by NSF, NASA, and the Powell Foundation.

The authors would like to thank Prof. G. Picci and Prof R. Frezza for their constant and enthusiastic support. Particular thanks go to Prof. C. I. Byrnes, Prof. C. Martin, Prof. S. S. Sastry and Prof. T. Taylor, who showed interest and enthusiasm at the time this work still had to be completed. 


\section{References}

Boothby, W. M. (1975). An Introduction to Differentiable Manifolds and Riemannian Geometry. New York: Academic Press.

Brockett, R. W. (1973). Lie theory and control systems defined on spheres. SIAM J. Applied Mathematics 25(2), 213-225.

Bullo, F. and R. M. Murray (1994). Proportional derivative (PD) control on matrix Lie groups: Introductory theory and examples. Submitted to the European Control Conference 1995.

Byrnes, C. I. and A. Isidori (1991). On the attitude stabilization of rigid spacecraft. Automatica 27, 87-95.

Crouch, P. (1984). Spacecraft attitude control and stabilization: Application of geometric control theory to rigid body models. IEEE Trans. Automatic Control AC-29, 321-331.

Koditschek, D. E. (1989). The application of total energy as a Lyapunov function for mechanical control systems. In P. S. K. J. E. Marsden and J. C. Simo (Eds.), Dynamics and Control of Multibody Systems, Volume 97, pp. 131-157. AMS.

Krishnan, H., M. Reyhanoglu, and H. McClamroch (1994). On the attitude stabilization of a rigid spacecraft using two control torques. Automatica 30(6), 1023-1027.

Marsden, J. E. (1992). Lectures on Mechanics. New York: Cambridge University Press.

Morin, P., C. Samson, J.-B. Pomet, and Z.-P. Jiang (1994). Time-varying feeback stabilization of the attitude of a rigid spacecraft with two controls. In IEEE Conference on Decision and Control, Lake Buena Vista, Florida, pp. 914-915.

Murray, R., Z. Li, and S. S. Sastry (1994). A Mathematical Introduction to Robotic Manipulation. Boca Raton, Florida: CRC Press.

Sastry, S. S. and M. Bodson (1989). Adaptive Control: Stability, Convergence and Robustness. Englewood Cliffs, NJ: Prentice Hall.

Slafer, L. I. and V. L. Seidenstucker (1991). Attitude and payload controlsystems for INTELSAT-VI. COMSAT Technical Review 21(1), 57100.

Swain, M. and M. Stricker (1991, November). Promising directions in active vision. Technical Report CS 91-27, University of Chicago. NSF Active Vision Workshop-August 5-7 1991. 
Tsiotras, P. and Y. M. Longuski (1994). Spin-axis stabilization of symmetrical spacecraft with 2 control torque. Systems \& Control Letters 23(6), 395-402.

Walsh, G., R. Montgomery, and S. S. Sastry (1994, June). Orientation control of the dynamic satellite. In American Control Conference, Baltimore, Maryland, pp. 138-142.

Walsh, G. and S. Sastry (1995, February). On reorienting rigid linked bodies using internal motions. IEEE Trans. Robotics and Automation. Appeared also in the 1991 IEEE Conference on Decision and Control.

Wen, J. T.-Y. and D. S. Bayard (1988). A new class of control laws for robotic manipulators. Part I: Non-adaptive case. Int. Journal of Control 47(5), 1361-1385.

Wen, J. T.-Y. and K. Kreutz-Delgado (1991). The attitude control problem. IEEE Trans. Automatic Control AC-36, 1148-1162. 


\section{A Stability proofs for the PD controllers}

We report here the stability proofs of the PD control laws in Section 2.

\section{A.1 Exponential convergence for the PD regulator (Proof of Theorem 3)}

Proof. Using the notations introduced in Section 2, we rewrite here the closed-loop system:

$$
\left\{\begin{aligned}
\dot{p} & =X_{p}^{1} v_{1}+X_{p}^{2} v_{2}, \\
\dot{v} & =\operatorname{dist}(\mathrm{p}, \mathrm{q}) \mathrm{K}_{\mathrm{p}}\left[\begin{array}{c}
\left\langle Y_{p}^{q}, X_{p}^{1}\right\rangle \\
\left\langle Y_{p}^{q}, X_{p}^{2}\right\rangle
\end{array}\right]-\mathrm{K}_{\mathrm{d}} \mathrm{v},
\end{aligned}\right.
$$

where $v=\left[\begin{array}{ll}v_{1} & v_{2}\end{array}\right]^{T}$. Let us define

$$
\operatorname{Cross} \triangleq \operatorname{dist}(\mathrm{p}, \mathrm{q})\left\langle\dot{\mathrm{p}}, \mathrm{Y}_{\mathrm{p}}^{\mathrm{q}}\right\rangle_{\mathrm{T}_{\mathrm{p}} \mathbb{S}^{2}}=\operatorname{dist}(\mathrm{p}, \mathrm{q})\left\langle\left[\begin{array}{l}
v_{1} \\
v_{2}
\end{array}\right],\left[\begin{array}{c}
\left\langle Y_{p}^{q}, X_{p}^{1}\right\rangle \\
\left\langle Y_{p}^{q}, X_{p}^{2}\right\rangle
\end{array}\right]\right\rangle
$$

Note that, for small $\epsilon$, the candidate Lyapunov function

$$
\begin{aligned}
W_{\text {skewed }} & \triangleq \frac{1}{2} \operatorname{dist}(\mathrm{p}, \mathrm{q})^{2}+\frac{1}{2}\|\mathrm{v}\|_{\mathrm{K}_{\mathrm{p}}^{-1}}^{2}-\epsilon \text { Cross } \\
& =W-\epsilon \text { Cross, }
\end{aligned}
$$

is positive definite, since $|\operatorname{Cross}| \leq \operatorname{dist}(\mathrm{p}, \mathrm{q})\|\mathrm{v}\|$. Indeed, recall that the standard steps performed in Section 2, equation (2.6), give

$$
\dot{W}=-\|v\|_{K_{p}^{-1} K_{d}}^{2} .
$$

We now need to show that $\dot{W}_{\text {skewed }}$ is definite negative. Taking the time derivative of the cross term

$$
\begin{aligned}
\frac{d}{d t} \operatorname{Cross}= & \frac{d}{d t}(\operatorname{dist}(\mathrm{p}, \mathrm{q}))\left\langle\dot{\mathrm{p}}, \mathrm{Y}_{\mathrm{p}}^{\mathrm{q}}\right\rangle \\
& +\operatorname{dist}(\mathrm{p}, \mathrm{q})\left(\left\langle\left[\begin{array}{c}
\dot{v}_{1} \\
\dot{v}_{2}
\end{array}\right],\left[\begin{array}{c}
\left\langle Y_{p}^{q}, X_{p 1}\right\rangle \\
\left\langle Y_{p}^{q}, X_{p 2}\right\rangle
\end{array}\right]\right\rangle+\left\langle\dot{\mathrm{p}}, \frac{\mathrm{d}}{\mathrm{dt}} \mathrm{Y}_{\mathrm{p}}^{\mathrm{q}}\right\rangle\right) \\
\triangleq & -\left\langle\dot{p}, Y_{p}^{q}\right\rangle^{2}+B_{1}+B_{2},
\end{aligned}
$$

where we used Lemma 1 in the last step. 
We can now easily compute a bound on $B_{1}$ :

$$
\begin{aligned}
B_{1}= & \operatorname{dist}(\mathrm{p}, \mathrm{q})^{2}\left\langle\mathrm{~K}_{\mathrm{p}}\left[\begin{array}{l}
\left\langle Y_{p}^{q}, X_{p}^{1}\right\rangle \\
\left\langle Y_{p}^{q}, X_{p}^{2}\right\rangle
\end{array}\right],\left[\begin{array}{l}
\left\langle Y_{p}^{q}, X_{p}^{1}\right\rangle \\
\left\langle Y_{p}^{q}, X_{p}^{2}\right\rangle
\end{array}\right]\right\rangle \\
& -\operatorname{dist}(\mathrm{p}, \mathrm{q})\left\langle\mathrm{K}_{\mathrm{d}}\left[\begin{array}{l}
v_{1} \\
v_{2}
\end{array}\right],\left[\begin{array}{l}
\left\langle Y_{p}^{q}, X_{p}^{1}\right\rangle \\
\left\langle Y_{p}^{q}, X_{p}^{2}\right\rangle
\end{array}\right]\right\rangle \\
\geq & \lambda_{\min }\left(K_{p}\right) \operatorname{dist}(\mathrm{p}, \mathrm{q})^{2}-\lambda_{\max }\left(\mathrm{K}_{\mathrm{d}}\right) \operatorname{dist}(\mathrm{p}, \mathrm{q})\|\dot{\mathrm{p}}\|
\end{aligned}
$$

where $\lambda_{\min }\left(K_{p}\right)$ is the minimum eigenvalue of $K_{p}$ and $\lambda_{\max }\left(K_{d}\right)$ the maximum.

The lower bounding of the third term entails more computations. Let $d=\operatorname{dist}(\mathrm{p}, \mathrm{q})$ and notice that

$$
Y_{p}^{q}=[\operatorname{vers}[p, q], p]=\frac{q-\langle p, q\rangle p}{\sin d}
$$

Recall $\dot{d}=-\left\langle\dot{p}, Y_{p}^{q}\right\rangle ;$ we have

$$
\begin{aligned}
\frac{d}{d t} Y_{p}^{q} & =\frac{(-\langle\dot{p}, q\rangle p-\langle p, q\rangle \dot{p}) \sin d-(q-\langle p, q\rangle p) \cos d \dot{d}}{\sin ^{2} d} \\
& =-\frac{\langle\dot{p}, q\rangle}{\sin d} p-\frac{\langle p, q\rangle}{\sin d} \dot{p}+\frac{\cos d}{\sin d}\left\langle\dot{p}, Y_{p}^{q}\right\rangle Y_{p}^{q} \\
& =-\frac{\langle\dot{p}, q\rangle}{\sin d} p-\frac{\cos d}{\sin d}\left(\dot{p}-\left\langle\dot{p}, Y_{p}^{q}\right\rangle Y_{p}^{q}\right)
\end{aligned}
$$

Substituting this expression into the definition of $B_{2}$ we have:

$$
\begin{aligned}
B_{2} & =d\left\langle\dot{p}, \frac{d}{d t} Y_{p}^{q}\right\rangle=-\frac{d \cos d}{\sin d}\left\langle\dot{p},\left(\dot{p}-\left\langle\dot{p}, Y_{p}^{q}\right\rangle Y_{p}^{q}\right)\right\rangle \\
& =-\frac{d \cos d}{\sin d}\left(\|\dot{p}\|^{2}-\left\langle\dot{p}, Y_{p}^{q}\right\rangle^{2}\right) .
\end{aligned}
$$

Notice that for $d \in[0, \pi / 2]$

$$
B_{2} \geq-\max _{[0, \pi / 2]}\left(\frac{d \cos d}{\sin d}\right)\|\dot{p}\|^{2} \geq-2\|\dot{p}\|^{2}
$$

while for $d \in[\pi / 2, \pi)$ the addendum $B_{2}$ is positive and it holds $B_{2} \geq 0$. Eventually:

$$
B_{2} \geq-2\|\dot{p}\|^{2}
$$


By and large equation (A.2) gives

$$
\frac{d}{d t} \operatorname{Cross} \geq \lambda_{\min }\left(K_{p}\right) \operatorname{dist}(\mathrm{p}, \mathrm{q})^{2}-\lambda_{\max }\left(\mathrm{K}_{\mathrm{d}}\right) \operatorname{dist}(\mathrm{p}, \mathrm{q})\|\dot{\mathrm{p}}\|-3\|\dot{\mathrm{p}}\|^{2},
$$

and we have for the candidate Lyapunov function

$$
\begin{aligned}
\dot{W}_{\text {skewed }}= & \dot{W}-\epsilon \frac{d}{d t} \operatorname{Cross} \\
\leq- & -\lambda_{\min }\left(K_{p}\right) \operatorname{dist}(\mathrm{p}, \mathrm{q})^{2}+\epsilon \lambda_{\max }\left(\mathrm{K}_{\mathrm{d}}\right) \operatorname{dist}(\mathrm{p}, \mathrm{q})\|\dot{\mathrm{p}}\| \\
& \quad-\|v\|_{K_{p}^{-1} K_{d}-3 \epsilon I_{2}}^{2},
\end{aligned}
$$

which makes the derivative of the Lyapunov function in equation (A.1) negative definite. Thus there exists a sufficiently small $\lambda>0$ such that

$$
\dot{W}_{\text {skewed }} \leq-\lambda W_{\text {skewed }}
$$

and the convergence is exponential.

Note that the previous proof can be slightly refined by adopting the slight modified computations described in the next subsection.

\section{A.2 Exponential convergence for the PD trajectory tracker (Proof of Theorem 5)}

In the following we will adopt the notations in Section 2. We start by stating some preliminary equalities: by differentiating expressions (2.8) and (2.9) and premultiplying by $\mathcal{R}^{T}$, we have

$$
\begin{aligned}
{[\omega, p] } & =\mathcal{R}^{T} \dot{q}-\dot{p} \\
{[\omega,[p, q]] } & =\left(\mathcal{R}-I_{3}\right) \frac{d}{d t}[p, q] .
\end{aligned}
$$

Note that the first expression determines uniquely $\omega_{\perp}=\omega-\omega_{p} p$ (the orthogonal projection of $\omega$ onto $T_{p} \mathbb{S}^{2}$ ), while $\omega_{p}$ is implicitly defined in the second.

We will first prove exponential stability and then prove expression (2.13).

Proof of Part I: Exponential Stability. Let $\dot{e}=\dot{p}-\mathcal{R}^{T} \dot{q}$ and consider the candidate Lyapunov function

$$
\begin{aligned}
W & \triangleq \frac{k_{p}}{2} \operatorname{dist}(\mathrm{p}, \mathrm{q})^{2}+\frac{1}{2}\|\dot{\mathrm{e}}\|^{2}-\epsilon \operatorname{dist}(\mathrm{p}, \mathrm{q})\left\langle\dot{\mathrm{e}}, \mathrm{Y}_{\mathrm{p}}^{\mathrm{q}}\right\rangle \\
& =W_{1}+\epsilon \mathrm{W}_{2},
\end{aligned}
$$


where the positive definite $W_{1}$ is skewed by the cross term $W_{2}$. For small $\epsilon$ the function $W$ is positive definite in $\left(\operatorname{dist}(p, q) \mathrm{Y}_{\mathrm{p}}^{\mathrm{q}}, \dot{\mathrm{e}}\right) \in\left(T_{p} \mathbb{S}^{2}\right)^{2}$. Differentiating with respect to time along the solutions of $\ddot{p}=u$ :

$$
\begin{aligned}
\frac{d}{d t} W_{1}= & -k_{p} \operatorname{dist}(\mathrm{p}, \mathrm{q})\left\langle\dot{\mathrm{e}}, \mathrm{Y}_{\mathrm{p}}^{\mathrm{q}}\right\rangle+\left\langle\dot{\mathrm{e}}, \mathrm{u}-\mathcal{R}^{\mathrm{T}} \ddot{\mathrm{q}}-\dot{\mathcal{R}}^{\mathrm{T}} \dot{\mathrm{q}}\right\rangle \\
= & -k_{p} \operatorname{dist}(\mathrm{p}, \mathrm{q})\left\langle\dot{\mathrm{e}}, \mathrm{Y}_{\mathrm{p}}^{\mathrm{q}}\right\rangle \\
& +\left\langle\dot{e}, k_{p} \operatorname{dist}(\mathrm{p}, \mathrm{q}) \mathrm{Y}_{\mathrm{p}}^{\mathrm{q}}-\mathrm{K}_{\mathrm{d}} \dot{\mathrm{e}}+\omega_{\mathrm{p}}[\dot{\mathrm{p}}, \mathrm{p}]-\dot{\mathcal{R}}^{\mathrm{T}} \dot{\mathrm{q}}\right\rangle \\
= & \left\langle\dot{e},-K_{d} \dot{e}+\omega_{p}[\dot{p}, p]-\dot{\mathcal{R}}^{T} \dot{q}\right\rangle \\
= & -\|\dot{e}\|_{K_{d}}^{2}+\left\langle\dot{e}, \omega_{p}[\dot{p}, p]-\dot{\mathcal{R}}^{T} \dot{q}\right\rangle,
\end{aligned}
$$

where all the steps performed are standard. If $\omega_{\perp}=\omega-\omega_{p} p$, we have

$$
\begin{aligned}
\left\langle\dot{e}, \omega_{p}[\dot{p}, p]-\dot{\mathcal{R}}^{T} \dot{q}\right\rangle & =\left\langle\dot{e}, \omega_{p}[\dot{p}, p]-\left[\mathcal{R}^{T} \dot{q}, \omega\right]\right\rangle \\
& =\left\langle\dot{e}, \omega_{p}[\dot{p}, p]-\omega_{p}\left[\mathcal{R}^{T} \dot{q}, p\right]-\left[\mathcal{R}^{T} \dot{q}, \omega_{\perp}\right]\right\rangle \\
& =\omega_{p}\langle\dot{e},[\dot{e}, p]\rangle-\left\langle\dot{e},\left[\mathcal{R}^{T} \dot{q}, \omega_{\perp}\right]\right\rangle=0,
\end{aligned}
$$

since in the first triple product $\dot{e}$ is repeated and in the second one the three vectors belong to the same plane $T_{p} \mathbb{S}^{2}$. Thus we have

$$
\frac{d}{d t} W_{1}=-\|\dot{e}\|_{K_{d}}^{2}
$$

so that, setting $\epsilon=0$, we have proved the Lyapunov stability of the close loop plant. Given this result, we can now follow the same steps as in the proof of Theorem 3 and exploit condition (2.12). Indeed we have:

$$
k_{p}>\frac{\|\dot{e}(0)\|^{2}}{\pi^{2}-\operatorname{dist}(\mathrm{p}(0), \mathrm{q}(0))^{2}} \quad \Longrightarrow \quad \operatorname{dist}(\mathrm{p}(\mathrm{t}), \mathrm{q}(\mathrm{t}))<\sqrt{2 \mathrm{~W}_{1}(0)}<\pi,
$$

proving that, for all $t$, dist $(\mathrm{p}(\mathrm{t}), \mathrm{q}(\mathrm{t}))$ is bounded away from $\pi$; we will need this fact later. Now we go on to differentiate $W_{2}$ :

$$
\begin{aligned}
\frac{d}{d t} W_{2} & =\left\langle\dot{e}, Y_{p}^{q}\right\rangle^{2}-\operatorname{dist}(\mathrm{p}, \mathrm{q})\left\langle\ddot{\mathrm{e}}, \mathrm{Y}_{\mathrm{p}}^{\mathrm{q}}\right\rangle-\operatorname{dist}(\mathrm{p}, \mathrm{q})\left\langle\dot{\mathrm{e}}, \frac{\mathrm{d}}{\mathrm{dt}} \mathrm{Y}_{\mathrm{p}}^{\mathrm{q}}\right\rangle \\
& \leq\|\dot{e}\|^{2}+B_{1}+B_{2} .
\end{aligned}
$$

To simplify the notation let $d=\operatorname{dist}(\mathrm{p}, \mathrm{q})$. Substituting the control law in $(2.11)$ :

$$
\begin{aligned}
B_{1} & =-d\left\langle k_{p} d Y_{p}^{q}-K_{d} \dot{e}, Y_{p}^{q}\right\rangle-d\left\langle\omega_{p}[\dot{p}, p]-\dot{\mathcal{R}}^{T} \dot{q}, Y_{p}^{q}\right\rangle \\
& =-k_{p} d^{2}+d\left\langle K_{d} \dot{e}, Y_{p}^{q}\right\rangle-d\left\langle[\dot{e}, p]-\left[\mathcal{R}^{T} \dot{q}, \omega_{\perp}\right], Y_{p}^{q}\right\rangle .
\end{aligned}
$$


Now, note that $\|[\dot{e}, p]\| \leq\|\dot{e}\|$, and $\left\|\left[\mathcal{R}^{T} \dot{q}, \omega_{\perp}\right]\right\| \leq\|\dot{q}\| \cdot\left\|\omega_{\perp}\right\|=\|\dot{q}\| \cdot\|\dot{e}\|$ by equation (A.3). Therefore we have the bound

$$
\begin{aligned}
B_{1} & \leq-k_{p} d^{2}+\lambda_{\max }\left(K_{d}\right) d\|\dot{e}\|+d\|\dot{e}\|+d\|\dot{e}\| \cdot\|\dot{q}\| \\
& =-k_{p} d^{2}+\left(1+\lambda_{\max }\left(K_{d}\right)+\|\dot{q}\|\right) d\|\dot{e}\| .
\end{aligned}
$$

Regarding $B_{2}$ we have

$$
\begin{aligned}
\frac{d}{d t} Y_{p}^{q} & =\frac{d}{d t} \frac{q-\langle p, q\rangle p}{\sin (d)}=\frac{d}{d t} \frac{q-\cos (d) p}{\sin (d)} \\
& =\frac{\sin (d)(\dot{q}-\cos (d) \dot{p}+\sin (d) \dot{d} p)-(q-\cos (d) p) \cos (d) \dot{d}}{\sin (d)^{2}} \\
& =\frac{\dot{q}-\cos (d) \dot{p}}{\sin (d)}-\left\langle\dot{e}, Y_{p}^{q}\right\rangle p+\frac{\cos (d)}{\sin (d)}\left\langle\dot{e}, Y_{p}^{q}\right\rangle Y_{p}^{q} \\
& =-\left\langle\dot{e}, Y_{p}^{q}\right\rangle p-\frac{\cos (d)}{\sin (d)}\left(\dot{e}-\left\langle\dot{e}, Y_{p}^{q}\right\rangle\right)+\frac{I_{3}-\cos (d) \mathcal{R}^{T}}{\sin (d)} \dot{q} .
\end{aligned}
$$

From the definition of $\mathcal{R}$ in equation (2.7) we have

$$
\frac{I_{3}-\cos (d) \mathcal{R}^{T}}{\sin (d)}=\frac{1-\cos (d)}{\sin (d)}\left(I_{3}-(\operatorname{vers}[p, q] \times)^{2}\right)+\cos (d)(\operatorname{vers}[p, q] \times),
$$

so that, interpreting the matrices above, we have

$$
\left\|\frac{I_{3}-\cos (d) \mathcal{R}^{T}}{\sin (d)}\right\|_{2} \leq \max _{d}\left|\frac{1-\cos (d)}{\sin (d)}\right|+1
$$

Plugging in the this bound in the definition of $B_{2}$ we obtain

$$
\begin{aligned}
B_{2} & =-d\left\langle\dot{e}, \frac{d}{d t} Y_{p}^{q}\right\rangle \\
& =-d\left\langle\dot{e},-\frac{\cos (d)}{\sin (d)}\left(\dot{e}-\left\langle\dot{e}, Y_{p}^{q}\right\rangle\right)+\frac{I_{3}-\cos (d) \mathcal{R}^{T}}{\sin (d)} \dot{q}\right\rangle \\
& \leq \max _{d}\left|\frac{d \cos (d)}{\sin (d)}\right|\|\dot{e}\|^{2}+\left(\max _{d}\left|\frac{1-\cos (d)}{\sin (d)}\right|+1\right)\|\dot{q}\| d\|\dot{e}\| .
\end{aligned}
$$

Recall now that $d=\operatorname{dist}(\mathrm{p}, \mathrm{q})$ is bounded away from $\pi$. Thus there exist two positive constants $M_{1}$ and $M_{2}$ such that

$$
\max _{d}\left|\frac{d \cos (d)}{\sin (d)}\right|<M_{1} \text { and } \max _{d}\left|\frac{1-\cos (d)}{\sin (d)}\right|<M_{2},
$$


for all times $t$. Eventually we have

$$
B_{2} \leq M_{1}\|\dot{e}\|^{2}+\|\dot{q}\|\left(M_{2}+1\right) \operatorname{dist}(\mathrm{p}, \mathrm{q})\|\dot{\mathrm{e}}\| .
$$

Substituting $B_{1}$ and $B_{2}$ into equation (A.7), we have

$$
\begin{aligned}
\frac{d}{d t} W_{2} \leq & -k_{p} \operatorname{dist}(\mathrm{p}, \mathrm{q})^{2}+\left(1+\mathrm{M}_{1}\right)\|\dot{\mathrm{e}}\|^{2} \\
& +\left(1+\lambda_{\max }\left(K_{d}\right)+\left(2+M_{2}\right)\|\dot{q}\|\right) \operatorname{dist}(\mathrm{p}, \mathrm{q})\|\dot{\mathrm{e}}\| .
\end{aligned}
$$

Therefore our Lyapunov function $W$ in equation (A.5) satisfies

$$
\begin{aligned}
\frac{d}{d t} W= & \frac{d}{d t} W_{1}+\epsilon \frac{d}{d t} W_{2} \\
\leq & -\|\dot{e}\|_{K_{d}}^{2}+\epsilon \frac{d}{d t} W_{2} \\
\leq & -\epsilon k_{p} \operatorname{dist}(\mathrm{p},) \mathrm{q}^{2}-\left(\lambda_{\min }\left(\mathrm{K}_{\mathrm{d}}\right)-\epsilon\left(1+\mathrm{M}_{1}\right)\right)\|\dot{\mathrm{e}}\|^{2} \\
& +\epsilon\left(1+\lambda_{\max }\left(K_{d}\right)+\left(2+M_{2}\right)\|\dot{q}\|\right) \operatorname{dist}(\mathrm{p}, \mathrm{q})\|\dot{\mathrm{e}}\| \\
= & -\left[\begin{array}{cc}
\operatorname{dist}(\mathrm{p}, \mathrm{q}) \\
\|\dot{e}\|
\end{array}\right]^{T}\left[\begin{array}{ll}
\epsilon k_{p} & \epsilon * \\
\epsilon * & \lambda_{\min }\left(K_{d}\right)-\epsilon\left(1+M_{1}\right)
\end{array}\right]\left[\begin{array}{c}
\operatorname{dist}(\mathrm{p}, \mathrm{q}) \\
\|\dot{e}\|
\end{array}\right]
\end{aligned}
$$

where

$$
*=-\frac{1}{2}\left(1+\lambda_{\max }\left(K_{d}\right)+\left(2+M_{2}\right)\|\dot{q}\|\right) .
$$

Recalling that $\|\dot{q}\|$ is bounded by assumption, $\frac{d}{d t} W$ is negative definite for sufficiently small $\epsilon$ in $\left(\operatorname{dist}(\mathrm{p}, \mathrm{q}) \mathrm{Y}_{\mathrm{p}}^{\mathrm{q}}, \dot{\mathrm{e}}\right) \in\left(T_{p} \mathbb{S}^{2}\right)^{2}$. This proves exponential stability.

Proof of Part II: Expression (2.13). Multiply equation (A.4) by $Y_{p}^{q}$ and then apply the triple product rule to obtain

$$
\omega_{p}=\left\langle\frac{I_{3}-\mathcal{R}^{T}}{\sin (\operatorname{dist}(\mathrm{p}, \mathrm{q}))} Y_{p}^{q}, \frac{d}{d t}[p, q]\right\rangle .
$$

From the definition of $\mathcal{R}$ in equation (2.7) we have

$$
\frac{I_{3}-\mathcal{R}^{T}}{\sin (\operatorname{dist}(\mathrm{p}, \mathrm{q}))}=(\operatorname{vers}[p, q] \times)-\tan \left(\frac{1}{2} \operatorname{dist}(\mathrm{p}, \mathrm{q})\right)(\operatorname{vers}[\mathrm{p}, \mathrm{q}] \times)^{2}
$$

so that

$$
\frac{I_{3}-\mathcal{R}^{T}}{\sin (\operatorname{dist}(\mathrm{p}, \mathrm{q}))} Y_{p}^{q}=-p+\tan \left(\frac{1}{2} \operatorname{dist}(\mathrm{p}, \mathrm{q})\right) \mathrm{Y}_{\mathrm{p}}^{\mathrm{q}}
$$

The result is now straightforward. 


\section{B Stability proofs for the adaptive control laws}

We report here the stability proofs of adaptive control laws presented in Section 4.

\section{B.1 Adaptive Regulation of the kinematic model (Proof of Theorem 10)}

Proof. We rewrite here the closed-loop system for sake of completeness:

$$
\begin{aligned}
& \dot{\pi}_{0}=\operatorname{dist}\left(\pi_{0}, \mathrm{q}\right) \mathrm{K}_{\mathrm{p}}\left[\begin{array}{c}
\left\langle Y_{\pi_{0}}^{q}, \pi_{1}\right\rangle \\
\left\langle Y_{\pi_{0}}^{q}, \pi_{2}\right\rangle
\end{array}\right]+\left[\begin{array}{cc}
0 & 1 \\
-1 & 0
\end{array}\right]\left[\begin{array}{cc}
J_{1} & 0 \\
0 & J_{2}
\end{array}\right]^{-1}\left[\begin{array}{c}
\left\langle\tilde{m}_{0}, \pi_{1}\right\rangle \\
\left\langle\tilde{m}_{0}, \pi_{2}\right\rangle
\end{array}\right], \\
& \dot{\hat{m}}_{0}=-\operatorname{dist}\left(\pi_{0}, \mathrm{q}\right) \operatorname{\Gamma RJ}^{-1} \mathrm{R}^{\mathrm{T}} \operatorname{vers}\left[\pi_{0}, \mathrm{q}\right],
\end{aligned}
$$

where $m_{0}$ is the total constant angular momentum, $\widehat{m}_{0}$ its estimate and $\tilde{m}_{0}=m_{0}-\widehat{m}_{0}$ the estimation error.

Let $W$ be our candidate Lyapunov function:

$$
W=\frac{1}{2} \operatorname{dist}\left(\pi_{0}, \mathrm{q}\right)^{2}+\frac{1}{2}\left\|\tilde{\mathrm{m}}_{0}\right\|_{\Gamma^{-1}}^{2},
$$

where $\Gamma=\Gamma^{T}>0$ is the adaptation gain.

Following the steps in proof of Theorem 2, we have

$$
\begin{aligned}
\dot{W}= & -\operatorname{dist}\left(\pi_{0}, \mathrm{q}\right)^{2}\left\langle\mathrm{~K}_{\mathrm{p}}\left[\begin{array}{l}
\left\langle Y_{\pi_{0}}^{q}, \pi_{1}\right\rangle \\
\left\langle Y_{\pi_{0}}^{q}, \pi_{2}\right\rangle
\end{array}\right],\left[\begin{array}{c}
\left\langle Y_{\pi_{0}}^{q}, \pi_{1}\right\rangle \\
\left\langle Y_{\pi_{0}}^{q}, \pi_{2}\right\rangle
\end{array}\right]\right\rangle-\left\langle\tilde{\mathrm{m}}_{0}, \Gamma^{-1} \frac{\mathrm{d}}{\mathrm{dt}} \widehat{\mathrm{m}}_{0}\right\rangle \\
& -\operatorname{dist}\left(\pi_{0}, \mathrm{q}\right)\left\langle\left[\begin{array}{c}
\left\langle Y_{\pi_{0}}^{q}, \pi_{1}\right\rangle \\
\left\langle Y_{\pi_{0}}^{q}, \pi_{2}\right\rangle
\end{array}\right],\left[\begin{array}{cc}
0 & 1 \\
-1 & 0
\end{array}\right]\left[\begin{array}{cc}
J_{1}^{-1} & 0 \\
0 & J_{2}^{-1}
\end{array}\right]\left[\begin{array}{c}
\left\langle\tilde{m}_{0}, \pi_{1}\right\rangle \\
\left\langle\tilde{m}_{0}, \pi_{2}\right\rangle
\end{array}\right]\right\rangle \\
\leq & -\lambda_{\min }\left(K_{p}\right) \operatorname{dist}\left(\pi_{0}, \mathrm{q}\right)^{2}-\left\langle\tilde{\mathrm{m}}_{0}, \Gamma^{-1} \frac{\mathrm{d}}{\mathrm{dt}} \widehat{\mathrm{m}}_{0}\right\rangle \\
& -\operatorname{dist}\left(\pi_{0}, \mathrm{q}\right)\left\langle\left[\begin{array}{cc}
J_{1}^{-1} & 0 \\
0 & J_{2}^{-1}
\end{array}\right]\left[\begin{array}{cc}
0 & -1 \\
1 & 0
\end{array}\right]\left[\begin{array}{l}
\left\langle Y_{\pi_{0}}^{q}, \pi_{1}\right\rangle \\
\left\langle Y_{\pi_{0}}^{q}, \pi_{2}\right\rangle
\end{array}\right],\left[\begin{array}{l}
\left\langle R^{T} \tilde{m}_{0}, e_{1}\right\rangle \\
\left\langle R^{T} \tilde{m}_{0}, e_{2}\right\rangle
\end{array}\right]\right\rangle .
\end{aligned}
$$

Note that with respect to the basis $\left\{\pi_{1}, \pi_{2}\right\}$ of $T_{\pi_{0}} \mathbb{S}^{2}$ it holds

$$
Y_{\pi_{0}}^{q}=\operatorname{vers}\left[\begin{array}{l}
\left\langle\pi_{1}, q\right\rangle \\
\left\langle\pi_{2}, q\right\rangle
\end{array}\right]
$$

and

$$
\left[\begin{array}{cc}
0 & -1 \\
1 & 0
\end{array}\right] \text { vers }\left[\begin{array}{c}
\left\langle\pi_{1}, q\right\rangle \\
\left\langle\pi_{2}, q\right\rangle
\end{array}\right]=\left[\begin{array}{c}
\left\langle\operatorname{vers}\left[\pi_{0}, q\right], \pi_{1}\right\rangle \\
\left\langle\operatorname{vers}\left[\pi_{0}, q\right], \pi_{2}\right\rangle
\end{array}\right]=\left[\begin{array}{l}
\left\langle R^{T} \operatorname{vers}\left[\pi_{0}, q\right], e_{1}\right\rangle \\
\left\langle R^{T} \operatorname{vers}\left[\pi_{0}, q\right], e_{2}\right\rangle
\end{array}\right]
$$


Substituting, we can then notice:

$$
\begin{aligned}
\dot{W} \leq & -\lambda_{\min }\left(K_{p}\right) \operatorname{dist}\left(\pi_{0}, \mathrm{q}\right)^{2}-\left\langle\tilde{\mathrm{m}}_{0}, \Gamma^{-1} \frac{\mathrm{d}}{\mathrm{dt}} \widehat{\mathrm{m}}_{0}\right\rangle \\
& -\operatorname{dist}\left(\pi_{0}, \mathrm{q}\right)\left\langle\left[\begin{array}{cc}
J_{1}^{-1} & 0 \\
0 & J_{2}^{-1}
\end{array}\right]\left[\begin{array}{l}
\left\langle R^{T} \operatorname{vers}\left[\pi_{0}, q\right], e_{1}\right\rangle \\
\left\langle R^{T} \operatorname{vers}\left[\pi_{0}, q\right], e_{2}\right\rangle
\end{array}\right],\left[\begin{array}{c}
\left\langle R^{T} \tilde{m}_{0}, e_{1}\right\rangle \\
\left\langle R^{T} \tilde{m}_{0}, e_{2}\right\rangle
\end{array}\right]\right\rangle_{\mathbb{R}^{2}} \\
\leq & -\lambda_{\min }\left(K_{p}\right) \operatorname{dist}\left(\pi_{0}, \mathrm{q}\right)^{2}-\left\langle\tilde{\mathrm{m}}_{0}, \Gamma^{-1} \frac{\mathrm{d}}{\mathrm{dt}} \widehat{\mathrm{m}}_{0}\right\rangle \\
& -\operatorname{dist}\left(\pi_{0}, \mathrm{q}\right)\left\langle\mathrm{J}^{-1} \mathrm{R}^{\mathrm{T}} \operatorname{vers}\left[\pi_{0}, \mathrm{q}\right], \mathrm{R}^{\mathrm{T}} \tilde{\mathrm{m}}_{0}\right\rangle_{\mathbb{R}^{3}}
\end{aligned}
$$

and picking

$$
\Gamma^{-1} \frac{d}{d t} \widehat{m}_{0}=\operatorname{dist}\left(\pi_{0}, \mathrm{q}\right) \mathrm{RJ}^{-1} \mathrm{R}^{\mathrm{T}} \operatorname{vers}\left[\pi_{0}, \mathrm{q}\right],
$$

the last two addenda cancel. Eventually

$$
\dot{W} \leq-\lambda_{\min }\left(K_{p}\right) \operatorname{dist}\left(\pi_{0}, \mathrm{q}\right)^{2} .
$$

By Lasalle's Principle the asymptotic stability of the closed-loop spacecraft system follows:

$$
\lim _{t \rightarrow \infty} \operatorname{dist}\left(\pi_{0}(\mathrm{t}), \mathrm{q}\right)=0
$$

and consequently

$$
\lim _{t \rightarrow \infty} \frac{d}{d t} \widehat{m}_{0}=0
$$

\section{B.2 Adaptive Regulation of the dynamic model (Proof of Theorem 13)}

Proof. We rewrite here the closed-loop system for sake of completeness:

$$
\begin{aligned}
\dot{\pi}_{0} & =\left[\begin{array}{cc}
0 & 1 \\
-1 & 0
\end{array}\right]\left[\begin{array}{l}
\omega_{1} \\
\omega_{2}
\end{array}\right] \\
{\left[\begin{array}{l}
J_{1} \dot{\omega}_{1} \\
J_{2} \dot{\omega}_{2}
\end{array}\right] } & =\left[\begin{array}{c}
\left.\left\langle R^{T} \tilde{m}_{0}, \omega\right], e_{1}\right\rangle \\
\left\langle\left[R^{T} \tilde{m}_{0}, \omega\right], e_{2}\right\rangle
\end{array}\right]+\operatorname{dist}\left(\pi_{0}, \mathrm{q}\right) \mathrm{K}_{\mathrm{p}}\left[\begin{array}{c}
-\left\langle Y_{\pi_{0}}^{q}, \pi_{2}\right\rangle \\
\left\langle Y_{\pi_{0}}^{q}, \pi_{1}\right\rangle
\end{array}\right]-\mathrm{K}_{\mathrm{d}}\left[\begin{array}{l}
\omega_{1} \\
\omega_{2}
\end{array}\right] \\
\frac{d}{d t} \widehat{m}_{0} & =\Gamma R(\omega \times)\left[\begin{array}{c}
K_{p}^{-1}\left[\begin{array}{l}
\omega_{1} \\
\omega_{2}
\end{array}\right] \\
0
\end{array}\right]-\epsilon \operatorname{dist}\left(\pi_{0}, \mathrm{q}\right) \mathrm{R}(\omega \times) \mathrm{J}^{-1} \operatorname{vers}\left[\mathrm{e}_{0}, \mathrm{R}^{\mathrm{T}} \mathrm{q}\right]
\end{aligned}
$$


Define the candidate Lyapunov function:

$$
\begin{aligned}
W \triangleq & \frac{1}{2} \operatorname{dist}\left(\pi_{0}, \mathrm{q}\right)^{2}+\left\langle\left[\begin{array}{l}
\omega_{1} \\
\omega_{2}
\end{array}\right], \mathrm{K}_{\mathrm{p}}^{-1}\left[\begin{array}{cc}
J_{1} & 0 \\
0 & J_{2}
\end{array}\right]\left[\begin{array}{l}
\omega_{1} \\
\omega_{2}
\end{array}\right]\right\rangle \\
& -\epsilon \operatorname{dist}\left(\pi_{0}, \mathrm{q}\right)\left\langle\left[\begin{array}{l}
\omega_{1} \\
\omega_{2}
\end{array}\right],\left[\begin{array}{c}
-\left\langle Y_{\pi_{0}}^{q}, \pi_{2}\right\rangle \\
\left\langle Y_{\pi_{0}}^{q}, \pi_{1}\right\rangle
\end{array}\right]\right\rangle+\left\|\tilde{\mathrm{m}}_{0}\right\|_{\Gamma^{-1}+\mathrm{I}_{3}}^{2} \\
\equiv & W_{\text {old }}+\left\|\tilde{m}_{0}\right\|_{\Gamma^{-1}+I_{3}}^{2} .
\end{aligned}
$$

If $\tilde{m}_{0}=0$, we know that $\dot{W}_{\text {old }} \leq-\lambda W_{\text {old }}$ for some small $\lambda>0$. Here instead, in the derivative of $W_{\text {old }}$ there are two additional terms due to the drift. We have:

$$
\begin{aligned}
\dot{W} \leq & -\lambda W_{\text {old }}+\left\langle\left[\begin{array}{l}
\omega_{1} \\
\omega_{2}
\end{array}\right], K_{p}^{-1}\left[\begin{array}{l}
\left\langle\left[R^{T} \tilde{m}_{0}, \omega\right], e_{1}\right\rangle \\
\left\langle\left[R^{T} \tilde{m}_{0}, \omega\right], e_{2}\right\rangle
\end{array}\right]\right\rangle-\left\langle\tilde{m}_{0},\left(\Gamma^{-1}+I_{3}\right) \frac{d}{d t} \widehat{m}_{0}\right\rangle \\
& -\epsilon \operatorname{dist}\left(\pi_{0}, q\right)\left\langle\left[\begin{array}{cc}
J_{1} & 0 \\
0 & J_{2}
\end{array}\right]^{-1}\left[\begin{array}{l}
\left\langle\left[R^{T} \tilde{m}_{0}, \omega\right], e_{1}\right\rangle \\
\left\langle\left[R^{T} \tilde{m}_{0}, \omega\right], e_{2}\right\rangle
\end{array}\right],\left[\begin{array}{c}
-\left\langle Y_{\pi_{0}}^{q}, \pi_{2}\right\rangle \\
\left\langle Y_{\pi_{0}}^{q}, \pi_{1}\right\rangle
\end{array}\right]\right\rangle .
\end{aligned}
$$

Note that the second addendum satisfies:

$$
\begin{aligned}
& \left\langle\left[\begin{array}{l}
\omega_{1} \\
\omega_{2}
\end{array}\right], K_{p}^{-1}\left[\begin{array}{l}
\left\langle\left[R^{T} \tilde{m}_{0}, \omega\right], e_{1}\right\rangle \\
\left\langle\left[R^{T} \tilde{m}_{0}, \omega\right], e_{2}\right\rangle
\end{array}\right]\right\rangle=\left\langle K_{p}^{-1}\left[\begin{array}{l}
\omega_{1} \\
\omega_{2}
\end{array}\right],\left[\begin{array}{l}
\left\langle\left[R^{T} \tilde{m}_{0}, \omega\right], e_{1}\right\rangle \\
\left\langle\left[R^{T} \tilde{m}_{0}, \omega\right], e_{2}\right\rangle
\end{array}\right]\right\rangle_{\mathbb{R}^{2}} \\
& =\left\langle\left[\begin{array}{c}
K_{p}^{-1}\left[\begin{array}{l}
\omega_{1} \\
\omega_{2}
\end{array}\right] \\
0
\end{array}\right],\left[R^{T} \tilde{m}_{0}, \omega\right]\right\rangle_{\mathbb{R}^{3}} \\
& =\left\langle\tilde{m}_{0}, R(\omega \times)\left[\begin{array}{c}
K_{p}^{-1}\left[\begin{array}{l}
\omega_{1} \\
\omega_{2}
\end{array}\right] \\
0
\end{array}\right]\right\rangle \text {. }
\end{aligned}
$$

Note that the fourth addendum satisfies, remember equation (B.1):

$$
\begin{gathered}
\left\langle\left[\begin{array}{cc}
J_{1} & 0 \\
0 & J_{2}
\end{array}\right]^{-1}\left[\begin{array}{l}
\left\langle\left[R^{T} \tilde{m}_{0}, \omega\right], e_{1}\right\rangle \\
\left\langle\left[R^{T} \tilde{m}_{0}, \omega\right], e_{2}\right\rangle
\end{array}\right],\left[\begin{array}{c}
-\left\langle Y_{\pi_{0}}^{q}, \pi_{2}\right\rangle \\
\left\langle Y_{\pi_{0}}^{q}, \pi_{1}\right\rangle
\end{array}\right]\right\rangle \\
\left.\left.=\left\langle\begin{array}{r}
\left\langle\left[R^{T} \tilde{m}_{0}, \omega\right], e_{1}\right\rangle \\
\left\langle\left[R^{T} \tilde{m}_{0}, \omega\right], e_{2}\right\rangle
\end{array}\right],\left[\begin{array}{cc}
J_{1} & 0 \\
0 & J_{2}
\end{array}\right]^{-1} \begin{array}{l}
\left\langle R^{T} \operatorname{vers}\left[\pi_{0}, q\right], e_{1}\right\rangle \\
\left\langle R^{T} \operatorname{vers}\left[\pi_{0}, q\right], e_{2}\right\rangle
\end{array}\right]\right\rangle_{\mathbb{R}^{2}} \\
=\left\langle\left[R^{T} \tilde{m}_{0}, \omega\right], J^{-1} R^{T} \operatorname{vers}\left[\pi_{0}, q\right]\right\rangle_{\mathbb{R}^{3}} \\
=\left\langle\tilde{m}_{0}, R(\omega \times) J^{-1} R^{T} \operatorname{vers}\left[\pi_{0}, q\right]\right\rangle .
\end{gathered}
$$

The two terms in the update law are chosen such as to render:

$$
\dot{W} \leq-\lambda W_{\text {old }},
$$


and by Lasalle's principle, the closed-loop trajectories of the system converge asymptotically to the largest invariant set contained in $\Omega=\Omega_{\text {old }}$. Hence

$$
\lim _{t \rightarrow \infty} \operatorname{dist}\left(\pi_{0}(\mathrm{t}), \mathrm{q}\right)=\lim _{\mathrm{t} \rightarrow \infty} \omega_{1}(\mathrm{t})=\lim _{\mathrm{t} \rightarrow \infty} \omega_{2}(\mathrm{t})=0 .
$$

